
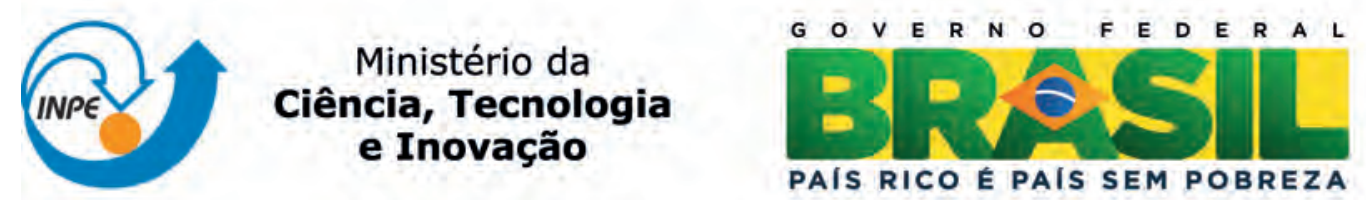

sid.inpe.br/mtc-m19/2011/12.05.16.29-RPQ

\title{
TOKAMAK EQUILIBRIA WITH STRONG TOROIDAL CURRENT DENSITY REVERSAL
}

\author{
Gerson Otto Ludwig \\ Paulo Rodrigues \\ João Pedro S. Bizarro
}

URL do documento original:

<http://urlib.net/8JMKD3MGP7W/3ATLNTP >

INPE

São José dos Campos

2011 


\section{PUBLICADO POR:}

Instituto Nacional de Pesquisas Espaciais - INPE

Gabinete do Diretor (GB)

Serviço de Informação e Documentação (SID)

Caixa Postal 515 - CEP 12.245-970

São José dos Campos - SP - Brasil

Tel.:(012) 3208-6923/6921

Fax: (012) 3208-6919

E-mail: pubtc@sid.inpe.br

\section{CONSELHO DE EDITORAÇÃO E PRESERVAÇÃO DA PRODUÇÃO INTELECTUAL DO INPE (RE/DIR-204): \\ Presidente:}

Marciana Leite Ribeiro - Serviço de Informação e Documentação (SID)

\section{Membros:}

Dr. Antonio Fernando Bertachini de Almeida Prado - Coordenação Engenharia e Tecnologia Espacial (ETE)

Dra Inez Staciarini Batista - Coordenação Ciências Espaciais e Atmosféricas (CEA)

Dr. Gerald Jean Francis Banon - Coordenação Observação da Terra (OBT)

Dr. Germano de Souza Kienbaum - Centro de Tecnologias Especiais (CTE)

Dr. Manoel Alonso Gan - Centro de Previsão de Tempo e Estudos Climáticos $(\mathrm{CPT})$

Dra Maria do Carmo de Andrade Nono - Conselho de Pós-Graduação

Dr. Plínio Carlos Alvalá - Centro de Ciência do Sistema Terrestre (CST)

\section{BIBLIOTECA DIGITAL:}

Dr. Gerald Jean Francis Banon - Coordenação de Observação da Terra (OBT)

Deicy Farabello - Centro de Previsão de Tempo e Estudos Climáticos (CPT)

\section{REVISÃO E NORMALIZAÇÃO DOCUMENTÁRIA:}

Marciana Leite Ribeiro - Serviço de Informação e Documentação (SID)

Yolanda Ribeiro da Silva Souza - Serviço de Informação e Documentação (SID)

EDITORAÇÃO ELETRÔNICA:

Vivéca Sant 'Ana Lemos - Serviço de Informação e Documentação (SID) 

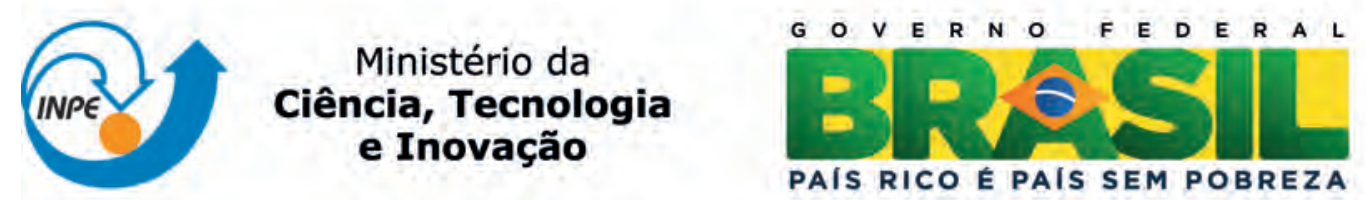

sid.inpe.br/mtc-m19/2011/12.05.16.29-RPQ

\title{
TOKAMAK EQUILIBRIA WITH STRONG TOROIDAL CURRENT DENSITY REVERSAL
}

\author{
Gerson Otto Ludwig \\ Paulo Rodrigues \\ João Pedro S. Bizarro
}

URL do documento original:

<http://urlib.net/8JMKD3MGP7W/3ATLNTP >

INPE

São José dos Campos

2011 
Dados Internacionais de Catalogação na Publicação (CIP)

\author{
Gonçalves, André Rodrigues. \\ R618r Tokamak equilibria with strong toroidal current density rever- \\ sal / Gerson Otto Ludwig \\ Paulo Rodrigues \\ João Pedro S. Bizarro. - São José dos Campos : INPE, 2011. \\ xxvi + 151 p. ; (sid.inpe.br/mtc-m19/2011/12.05.16.29-RPQ) \\ Dissertação (Mestrado em Meteorologia) - Instituto Nacional \\ de Pesquisas Espaciais, São José dos Campos, 2011. \\ Orientador : Dr. Enio Bueno Pereira. \\ 1. Previsão de vento. 2. Refinamento estatístico. 3. Camada \\ limite atmosférica. 4. Energia eólica. I.Título.
}

CDU 621.548:551

Copyright (c) 2011 do MCT/INPE. Nenhuma parte desta publicação pode ser reproduzida, armazenada em um sistema de recuperação, ou transmitida sob qualquer forma ou por qualquer meio, eletrônico, mecânico, fotográfico, reprográfico, de microfilmagem ou outros, sem a permissão escrita do INPE, com exceção de qualquer material fornecido especificamente com o propósito de ser entrado e executado num sistema computacional, para o uso exclusivo do leitor da obra.

Copyright (c) 2011 by MCT/INPE. No part of this publication may be reproduced, stored in a retrieval system, or transmitted in any form or by any means, electronic, mechanical, photocopying, recording, microfilming, or otherwise, without written permission from INPE, with the exception of any material supplied specifically for the purpose of being entered and executed on a computer system, for exclusive use of the reader of the work. 


\section{TABLE OF CONTENTS}

Page

1. Introduction.............................................................. 1

2. Spectral representation of flux surfaces................................. 2

3. Plasma equilibrium in the magnetic islands............................... 4

4. Equilibrium calculation by matching moments.......................... 6

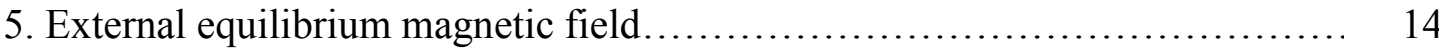

6. Magnetohydrodynamic stability analysis............................... 22

7. Discussion........................................................ 33 


\title{
Tokamak equilibria with strong toroidal current density reversal
}

\author{
G.O. Ludwig ${ }^{1 *}$ P. Rodrigues ${ }^{2}$, J.P.S. Bizarro ${ }^{2}$ \\ ${ }^{1}$ Laboratório Associado de Plasma, INPE, 12227-010 São José dos Campos, SP, Brasil. \\ ${ }^{2}$ Centro de Fusão Nuclear, Associação Euratom-IST, 1049-001 Lisboa, Portugal.
}

\begin{abstract}
The equilibrium of large magnetic islands in the core of a tokamak in conditions of strong toroidal current density reversal is investigated by a new method. The method uses distinct spectral representations to describe each simply connected region as well as the containing shell that substitutes for the external plasma region. Equivalent surface current densities are defined on the boundaries of the islands and on the thin shell, giving a straightforward formulation to the interaction between regions. The equilibrium of the islands-shell system is determined by matching moments of the Dirichlet boundary conditions. The magnetohydrodynamic stability against a class of tilting displacements is examined by means of an energy principle. It is found out that the symmetric islands are stable but the nonsymmetric system presents a bifurcation in the equilibrium.
\end{abstract}

PACS numbers: 28.52.Av Theory, design, and computerized simulation; 52.55.Fa Tokamaks, spherical tokamaks; 52.65.Kj Magnetohydrodynamic and fluid equation.

\section{Introduction}

A succession of experiments that spanned two decades $[1,2,3,4]$ have shown the feasibility of tokamak operation in the alternating current $(\mathrm{AC})$ regime. These configurations are closely related to the current-hole regime observed in large tokamak experiments $[5,6]$. Although the AC experiments in low temperature plasmas clearly show the formation of two large magnetic islands with opposite currents during the current reversal phase [7], the magnetic topology in the plasma core of current-hole experiments remains unresolved. It is not even clear if the toroidal current reverses in the core of current holes, as further experiments indicated a nearly zero current clamping in the central region [8]. Nevertheless, tokamak equilibria computations have shown that toroidal current reversal in the plasma core is compatible with the accuracy of present-day experiments [9]. The key to the seeming discrepancy lies in allowing for non-nested flux surfaces in the analysis of such plasmas, a phenomenon that is apparent in the AC operation of tokamaks but not always taken into account in current-hole studies.

Several theoretical papers addressed the problem of current reversal equilibria in tokamak plasmas $[10,11,12,13,14,15]$. The first paper in this list numerically examined the

\footnotetext{
*Corresponding author. E-mail address: ludwig@plasma.inpe.br
} 
current density reversal in force-free equilibria with non-nested flux surfaces [10]. A second series of papers analytically solved the Grad-Shafranov equation with a finite plasma pressure and a toroidal current density linearly dependent on the flux function, for circular and rectangular plasma cross-sections $[11,12,13]$. These results were numerically extended using the finite element method and considering parabolic profiles of the toroidal current density in terms of the flux function, for arbitrary plasma cross-sections [14]. Finally, numerical solutions of the recursively perturbed Grad-Shafranov equation for reversed equilibria were obtained without imposing particular models for the pressure and toroidal current density profiles [15]. In common, all these studies have shown that toroidal current reversal is necessarily accompanied by non-nested flux surfaces.

The present paper introduces a method to evaluate the equilibrium of magnetic islands in the core of a tokamak plasma with strong toroidal current reversal. The method is based on a simple model of the tokamak core described as follows. The core is enclosed by a magnetic flux surface (thin shell) which contains the set of idealized magnetic islands. The thin shell replaces the external region of the plasma, that may carry a substantial current density necessary for the equilibrium. Both the flux containing shell and the flux surfaces inside the islands are described in Section 2 using spectral representations with different poloidal angles defined for the shell centerline and for each simply connected region. This leads to a straightforward geometrical representation for an otherwise complex configuration of non-nested flux surfaces. A Fourier series expansion is used to describe the surface current density in the containing shell. The current densities in the islands may have large values and opposite signs but the current density between the containing shell and each island is neglected, resulting in a small, possibly zero total current in the plasma core. In Section 3 the plasma equilibrium inside each magnetic island is evaluated by a fourth-order radial series expansion of the variational moments solution to the Grad-Shafranov equation, compatible with but not limited to the large-aspect-ratio configuration of the islands. Then, the equilibrium of the system is determined in Section 4 by taking moments of the Dirichlet problem both on the containing shell and on the boundary of each contained island. The maintaining magnetic field on the containing shell (Neumann condition) is used in Section 5 to determine the currents in a simple set of external equilibrium coils. These coils do not correspond to the actual poloidal field coils system in the JT-60U tokamak, which is used as an example, but take a mere illustrative role of possible equilibria. Finally, a preliminary analysis of the magnetohydrodynamic stability of such system is carried out in Section 6 by evaluating the energy change associated with a small tilting displacement of the islands. In this way, it is demonstrated by example that a macroscopic, circuit-like approach can be used to analyze complex equilibria with small sets of islands in strongly-reversed-shear tokamak plasmas or in alternating current tokamaks. A summary and concluding remarks are given in Section 7.

\section{Spectral representation of flux surfaces}

The following parametric representation in cylindrical coordinates of the flux surfaces inside the magnetic islands includes Shafranov shift, elongation, triangularity and quadrangularity effects (higher order corrections can be included in a straightforward manner 
$[16]):$

$$
\begin{aligned}
R_{i}(\rho, \theta, t)= & R_{0, i}(\rho, t)+\rho \cos \theta \\
& +\frac{\rho^{2}}{a_{i}(t)}\left[S_{3, i}(\rho, t)(1-\cos \theta)+A_{3, i}(\rho, t) \sin \theta\right] \\
& -\rho\left[S_{2, i}(\rho, t)(1-\cos 2 \theta)-A_{2, i}(\rho, t) \sin 2 \theta\right] \\
& -\frac{\rho^{2}}{a_{i}(t)}\left[S_{3, i}(\rho, t)(1-\cos 3 \theta)-A_{3, i}(\rho, t) \sin 3 \theta\right], \\
\frac{Z_{i}(\rho, \theta, t)}{e_{i}(\rho, t)=} & \frac{Z_{0, i}(\rho, t)}{e_{i}(\rho, t)} \\
& +\rho \sin \theta+\frac{\rho^{2}}{a_{i}(t)}\left[A_{3, i}(\rho, t)(1-\cos \theta)-S_{3, i}(\rho, t) \sin \theta\right] \\
& -\rho\left[A_{2, i}(\rho, t)(1-\cos 2 \theta)+S_{2, i}(\rho, t) \sin 2 \theta\right] \\
& -\frac{\rho^{2}}{a_{i}(t)}\left[A_{3, i}(\rho, t)(1-\cos 3 \theta)+S_{3, i}(\rho, t) \sin 3 \theta\right] .
\end{aligned}
$$

Here $\rho=a_{i}(t)$ designates the edge of the island $i, R_{0, i}(\rho, t)$ is the major radius, $e_{i}(\rho, t)$ is the elongation coefficient, and $S_{n, i}(\rho, t), A_{n, i}(\rho, t)$ are the dimensionless symmetric and antisymmetric coefficients, respectively. Symmetry is defined with respect to the midplane $Z_{i}(\rho, 0, t)=Z_{0, i}(\rho, t)$. In general, the coefficients in the spectral representation are functions of the radial coordinate $\rho$ and time $t$. In the equilibria presented in this paper it is assumed that at least two islands, denoted by the subscripts $i$ and $j$, have been formed. The containing shell, which corresponds to the outermost flux surface defining the plasma core, has the simple representation:

$$
\begin{aligned}
R_{s}(\theta) & =R_{0, s}+a_{s} \cos \theta \\
Z_{s}(\theta) & =e_{s} a_{s} \sin \theta .
\end{aligned}
$$

The shell geometry is fixed by the parameters

$$
R_{0, s}=3.45 \mathrm{~m}, \quad a_{s}=0.82 \mathrm{~m}, \quad e_{s}=0.85
$$

which correspond to numerical equilibrium calculations performed for the JT-60U tokamak plasma core in conditions of strong toroidal current density reversal [17]. The main geometrical parameters of the JT-60U tokamak are: major radius $R_{0}=3.4 \mathrm{~m}$ and minor radius $a=1.0 \mathrm{~m}$. The toroidal surface current distribution in the containing shell is represented by a truncated Fourier series

$$
K_{T, s}(\theta, t)=\frac{1}{2 \pi h_{\theta, s}(\theta)}\left(I_{T, s}(t)+\sum_{m=1}^{n_{s}}\left[I_{m}^{(s)}(t) \cos m \theta+I_{m}^{(a)}(t) \sin m \theta\right]\right),
$$

where the poloidal scale factor is given as a function of the poloidal angle $\theta$ for the shell geometry

$$
h_{\theta, s}(\theta)=\sqrt{\left(\frac{\partial R_{s}}{\partial \theta}\right)^{2}+\left(\frac{\partial Z_{s}}{\partial \theta}\right)^{2}}=a_{s} \sqrt{1+\left(e_{s}^{2}-1\right) \cos ^{2} \theta} .
$$

The total current in the shell is calculated by the line integral

$$
I_{T, s}(t)=\int_{0}^{2 \pi} K_{T, s}(\theta, t) h_{\theta, s}(\theta) d \theta
$$




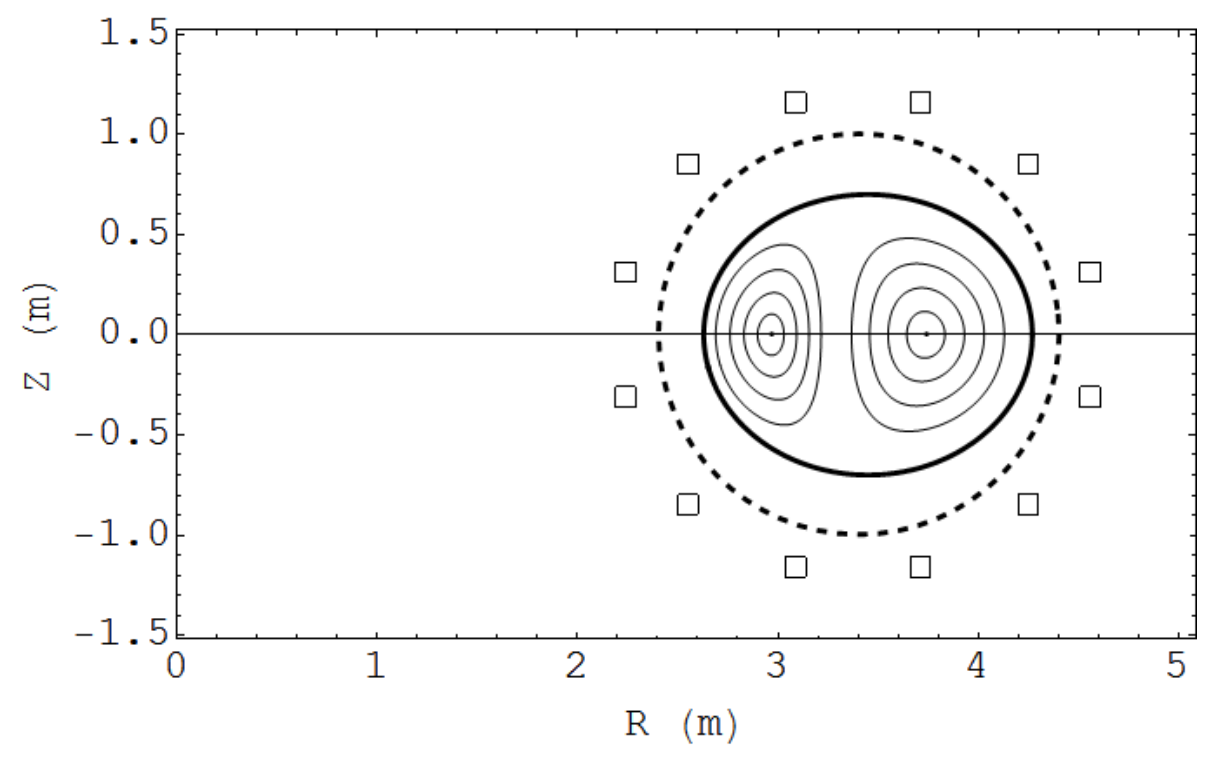

Figure 1: Set of two symmetric islands inside a containing shell representing the core of a large tokamak in a strong toroidal current density reversal configuration.

Figure 1 illustrates the flux surfaces inside two islands contained by a thin shell represented by the thick continuous line. This distribution of flux surfaces corresponds to the symmetric equilibrium calculated in Section 4. The dashed line corresponds to the main geometrical parameters of the JT-60U tokamak given above in the text. The small squares surrounding the torus illustrate the set of poloidal field coils used in Section 5 to establish the external equilibrium.

\section{Plasma equilibrium in the magnetic islands}

The Grad-Shafranov equation for the plasma equilibrium inside each magnetic island is solved by a Taylor series expansion to fourth-order in the radial coordinate $\rho$. A consistent power series expansion of the poloidal flux function near the magnetic axis of each island leads to the simplified spectral representation (neglecting higher than fourthorder corrections in the expansion of the poloidal flux function):

$$
\begin{aligned}
R_{i}(\rho, \theta, t) \cong & R_{m, i}(t)+\rho \cos \theta \\
& +\frac{\rho^{2}}{2}\left(R_{0, i}^{\prime \prime}(0, t)-4 S_{2, i}^{\prime}(0, t) \sin ^{2} \theta+2 A_{2, i}^{\prime}(0, t) \sin 2 \theta\right) \\
& -2 \frac{\rho^{3}}{a_{i}(t)}\left(S_{3, i}^{\prime}(0, t) \sin \theta-A_{3, i}^{\prime}(0, t) \cos \theta\right) \sin 2 \theta, \\
Z_{i}(\rho, \theta, t) \cong & Z_{m, i}(t)+\rho \kappa_{m, i}(t) \sin \theta \\
& +\frac{\rho^{2}}{2}\left[Z_{0, i}^{\prime \prime}(0, t)-2 \kappa_{m, i}(t)\left(S_{2, i}^{\prime}(0, t) \sin 2 \theta+A_{2, i}^{\prime}(0, t)(1-\cos 2 \theta)\right)\right] \\
& +\frac{\rho^{3}}{2}\left[e_{i}^{\prime \prime}(0, t)-8 \frac{\kappa_{m, i}(t)}{a_{i}(t)}\left(S_{3, i}^{\prime}(0, t) \cos \theta+A_{3, i}^{\prime}(0, t) \sin \theta\right) \cos \theta\right] \sin \theta \\
& -\rho^{4} e_{i}^{\prime \prime}(0, t)\left(S_{2, i}^{\prime}(0, t) \cos \theta+A_{2, i}^{\prime}(0, t) \sin \theta\right) \sin \theta,
\end{aligned}
$$


where the coordinates pair $R_{m, i}(t), Z_{m, i}(t)$ gives the position of the magnetic axis, $\kappa_{m, i}(t)$ corresponds to the elongation on axis, and the apostrophe indicates a partial derivative with respect to $\rho$. Now, according with the variational moments solution of the GradShafranov equation [18], the Fourier coefficients $R_{0, i}, Z_{0, i}, e_{i}, S_{2, i}, A_{2, i}, S_{3, i}$ and $A_{3, i}$ of the spectral representation satisfy a set of coupled Euler-Lagrange equations. Expanding the Euler-Lagrange equations in power series in $\rho$ and applying the geometrical boundary conditions at the edges $\rho=a_{i}(t)$ of the islands one obtains the lowest order solutions:

$$
\begin{aligned}
R_{0, i}(\rho, t) & \cong R_{m, i}(t)-\left[R_{m, i}(t)-R_{0, i}\left(a_{i}(t)\right)\right] \frac{\rho^{2}}{a_{i}^{2}(t)} \\
Z_{0, i}(\rho, t) & \cong Z_{m, i}(t)+\frac{2 \kappa_{m, i}(t)\left(3-\kappa_{m, i}^{2}(t)\right)}{3+\kappa_{m, i}^{2}(t)} A_{2, i}\left(a_{i}(t)\right) \frac{\rho^{2}}{a_{i}(t)} \\
e_{i}(\rho, t) & \cong \kappa_{m, i}(t)+\left[e_{i}\left(a_{i}(t)\right)-\kappa_{m, i}(t)\right] \frac{\rho^{2}}{a_{i}^{2}(t)} \\
S_{2, i}(\rho, t) & \cong S_{2, i}\left(a_{i}(t)\right) \frac{\rho}{a_{i}(t)} \\
A_{2, i}(\rho, t) & \cong A_{2, i}\left(a_{i}(t)\right) \frac{\rho}{a_{i}(t)} \\
S_{3, i}(\rho, t) & \cong S_{3, i}\left(a_{i}(t)\right) \frac{\rho}{a_{i}(t)} \\
A_{3, i}(\rho, t) & \cong A_{3, i}\left(a_{i}(t)\right) \frac{\rho}{a_{i}(t)}
\end{aligned}
$$

The radial position $R_{m, i}(t)$ of the magnetic axis is given in terms of both the major radius $R_{0, i}\left(a_{i}(t)\right)$ and of the symmetric triangularity coefficient $S_{2, i}\left(a_{i}(t)\right)$ at the boundary of the island, of the elongation $\kappa_{m, i}(t)$ at the magnetic axis, and of the second derivatives on axis of the plasma pressure profile $p_{i}^{\prime \prime}(0, t)=\partial^{2} p_{i}(0, t) / \partial \rho^{2}$ and of the toroidal current profile $I_{T, i}^{\prime \prime}(0, t)=\partial^{2} I_{T, i}(0, t) / \partial \rho^{2}$ by

$$
\begin{aligned}
R_{m, i}(t) \cong & \frac{R_{0, i}\left(a_{i}\right)}{2}-\frac{2 a_{i} S_{2, i}\left(a_{i}\right)}{1+3 \kappa_{m, i}^{2}} \\
& +\sqrt{\left(\frac{R_{0, i}\left(a_{i}\right)}{2}-\frac{2 a_{i} S_{2, i}\left(a_{i}\right)}{1+3 \kappa_{m, i}^{2}}\right)^{2}+\frac{\kappa_{m, i}^{2} a_{i}^{2}}{2\left(1+3 \kappa_{m, i}^{2}\right)}\left(1-\frac{8 \pi^{2}\left(1+\kappa_{m, i}^{2}\right)^{2} p_{i}^{\prime \prime}(0)}{\kappa_{m, i}^{2} \mu_{0} I_{T, i}^{\prime \prime}(0)^{2}}\right)},
\end{aligned}
$$

where the time dependence of the minor radius $a_{i}$, of the elongation $\kappa_{m, i}$, and of the derivatives $p_{i}^{\prime \prime}(0)$ and $I_{T, i}^{\prime \prime}(0)$ was omitted for simplicity. Likewise, the vertical position $Z_{m, i}(t)$ of the magnetic axis is given in terms of the major vertical position $Z_{0, i}\left(a_{i}(t)\right)$, of the antisymmetric triangularity coefficient $A_{2, i}\left(a_{i}(t)\right)$ and of the elongation $\kappa_{m, i}(t)$ by

$$
Z_{m, i}(t) \cong Z_{0, i}\left(a_{i}\right)-\frac{2 \kappa_{m, i}\left(3-\kappa_{m, i}^{2}\right)}{3+\kappa_{m, i}^{2}} A_{2, i}\left(a_{i}\right) a_{i}
$$


Finally, the magnetic axis elongation satisfies the high-order polynomial equation

$$
\begin{aligned}
& 3 \kappa_{m, i}\left(\kappa_{m, i}^{2}+3\right)^{2}\left\{2 a_{i}^{2} R_{m, i}\left[R_{0, i}\left(a_{i}\right)\left(3 \kappa_{m, i}^{4}+8 \kappa_{m, i}^{2}+1\right)-2 R_{m, i}\left(2 \kappa_{m, i}^{4}+13 \kappa_{m, i}^{2}+3\right)\right]\right. \\
& \left.+a_{i}^{4}\left(\kappa_{m, i}^{4}+6 \kappa_{m, i}^{2}+1\right)-4 R_{m, i}^{2}\left(R_{m, i}-R_{0, i}\left(a_{i}\right)\right)^{2}\left(3 \kappa_{m, i}^{4}+2 \kappa_{m, i}^{2}+3\right)\right\} \mu_{0} I_{T, i}^{\prime \prime}(0)^{2} \\
& +6 a_{i}^{2} R_{m, i}^{2}\left(\kappa_{m, i}^{2}+3\right)^{2}\left(\kappa_{m, i}^{4}+18 \kappa_{m, i}^{2}+5\right) e_{i}\left(a_{i}\right) \mu_{0} I_{T, i}^{\prime \prime}(0)^{2} \\
& -24 \pi^{2} a_{i}^{2} \kappa_{m, i}\left(\kappa_{m, i}^{2}+3\right)^{2}\left(\kappa_{m, i}^{2}+1\right)^{3}\left[a_{i}^{2}+8 R_{m, i}\left(R_{m, i}-R_{0, i}\left(a_{i}\right)\right)\right] p_{i}^{\prime \prime}(0) \\
& +12 a_{i} R_{m, i} \kappa_{m, i}\left(\kappa_{m, i}^{2}+3\right)^{2}\left[a_{i}^{2}\left(3 \kappa_{m, i}^{4}+2 \kappa_{m, i}^{2}-5\right)\right. \\
& \left.-8 R_{m, i}\left(R_{m, i}-R_{0, i}\left(a_{i}\right)\right)\left(3 \kappa_{m, i}^{4}+8 \kappa_{m, i}^{2}+3\right)\right] S_{2, i}\left(a_{i}\right) \mu_{0} I_{T, i}^{\prime \prime}(0)^{2} \\
& -192 \pi^{2} a_{i}^{3} R_{m, i} \kappa_{m, i}\left(\kappa_{m, i}^{2}+3\right)^{2}\left(\kappa_{m, i}^{2}+1\right)^{3} S_{2, i}\left(a_{i}\right) p_{i}^{\prime \prime}(0) \\
& -12 a_{i}^{2} R_{m, i}^{2} \kappa_{m, i}\left(\kappa_{m, i}^{2}+3\right)^{2}\left(19 \kappa_{m, i}^{4}+62 \kappa_{m, i}^{2}+23\right) S_{2, i}^{2}\left(a_{i}\right) \mu_{0} I_{T, i}^{\prime \prime}(0)^{2} \\
& -120 a_{i}^{2} R_{m, i}^{2} \kappa_{m, i}\left(\kappa_{m, i}^{2}+3\right)^{2}\left(\kappa_{m, i}^{4}-1\right) S_{3, i}\left(a_{i}\right) \mu_{0} I_{T, i}^{\prime \prime}(0)^{2} \\
& +a_{i}^{4} R_{m, i}^{2} \kappa_{m, i}\left(\kappa_{m, i}^{2}+3\right)^{2}\left(\kappa_{m, i}^{4}-1\right) \mu_{0} I_{T, i}^{\prime \prime}(0) I_{T, i}^{(4)}(0) \\
& -12 a_{i}^{2} R_{m, i}^{2} \kappa_{m, i}\left(\kappa_{m, i}^{2}-1\right)\left(25 \kappa_{m, i}^{6}+337 \kappa_{m, i}^{4}+555 \kappa_{m, i}^{2}+171\right) A_{2, i}^{2}\left(a_{i}\right) \mu_{0} I_{T, i}^{\prime \prime}(0)^{2}=0 .
\end{aligned}
$$

The elongation $\kappa_{m, i}$ on axis can be determined numerically using the Newton-Raphson formula

$$
\kappa_{m, i}^{(k+1)}=\kappa_{m, i}^{(k)}-\frac{f\left(\kappa_{m, i}^{(k)}\right)}{f^{\prime}\left(\kappa_{m, i}^{(k)}\right)},
$$

where $f\left(\kappa_{m, i}\right)=0$ designates the above polynomial equation for $\kappa_{m, i}$ obtained from the Euler-Lagrange equation for $e_{i}(\rho)$. The value of the elongation at the edge, $\kappa_{m, i}^{(0)}=e_{i}\left(a_{i}\right)$, is taken as trial solution, giving a very good approximation to the true root using the two or three steps Newton-Raphson method.

In this way, the plasma equilibrium in each island is described by seven edge-parameters, namely, $a_{i}, R_{0, i}\left(a_{i}\right), e_{i}\left(a_{i}\right), S_{2, i}\left(a_{i}\right), A_{2, i}\left(a_{i}\right), S_{3, i}\left(a_{i}\right)$ and $A_{3, i}\left(a_{i}\right)$, plus the plasma pressure profile and the toroidal current profile derivatives $p_{i}^{\prime \prime}(0), I_{T i}^{\prime \prime}(0)$ and $I_{T, i}^{(4)}(0)$ on the magnetic axis of the island. The major vertical position $Z_{0, i}(t)$ is irrelevant for the internal equilibrium of the island (only the difference $Z_{0, i}\left(a_{i}\right)-Z_{m, i}(t)$ matters) but it affects the external equilibrium. Of course, starting with this approximation a more precise solution of the Grad-Shafranov equation may be sought for, but the present one carries most of the relevant information about the islands equilibria in a very simple way.

\section{Equilibrium calculation by matching moments}

The shape of each island can be determined by matching moments of the Dirichlet conditions on the containing shell and on the islands' edges. The total poloidal flux $\Phi_{i}\left(a_{i}, \theta_{i}\right)$ on the edge of island $i$, for example, is given by the sum of the self-flux $\Phi_{i i}$ and the fluxes $\Phi_{j i}$ and $\Phi_{s i}$ produced by the island $j$ and by the shell $s$ on the boundary of $i$, respectively. These fluxes can be written in terms of the equivalent toroidal surface current densities on the respective boundaries:

$$
\begin{aligned}
\Phi_{i}\left(a_{i}, \theta_{i}\right)= & \mu_{0} \oint_{\ell_{i}} K_{T, i}\left(a_{i}, \theta_{i}^{\prime}\right) G\left(\theta_{i} ; \theta_{i}^{\prime}\right) d \ell\left(\theta_{i}^{\prime}\right)+\mu_{0} \oint_{\ell_{j}} K_{T, j}\left(a_{j}, \theta_{j}\right) G\left(\theta_{i} ; \theta_{j}\right) d \ell\left(\theta_{j}\right) \\
& +\mu_{0} \oint_{\ell_{s}} K_{T, s}\left(\theta_{s}\right) G\left(\theta_{i} ; \theta_{s}\right) d \ell\left(\theta_{s}\right) .
\end{aligned}
$$


Here $\theta_{i}, \theta_{j}$ and $\theta_{s}$ designate the poloidal angle variables along the boundaries of $i, j$ and $s$, respectively, with similar expressions for $\Phi_{j}\left(a_{j}, \theta_{j}\right)$ and $\Phi_{s}\left(a_{s}, \theta_{s}\right)$. The Green's function $G$ is given in terms of the complete elliptic integrals $K(m)$ and $E(m)$ by

$$
\left\{\begin{aligned}
G\left(\theta ; \theta^{\prime}\right) & =\sqrt{R(\theta) R\left(\theta^{\prime}\right)}\left(\frac{\left[2-m\left(\theta, \theta^{\prime}\right)\right] K\left[m\left(\theta, \theta^{\prime}\right)\right]-2 E\left[m\left(\theta, \theta^{\prime}\right)\right]}{\sqrt{m\left(\theta, \theta^{\prime}\right)}}\right) \\
m\left(\theta, \theta^{\prime}\right) & =\frac{4 R(\theta) R\left(\theta^{\prime}\right)}{\left[R(\theta)+R\left(\theta^{\prime}\right)\right]^{2}+\left[Z(\theta)-Z\left(\theta^{\prime}\right)\right]^{2}} \quad(0 \leqslant m \leqslant 1)
\end{aligned}\right.
$$

where $R(\theta)=R(a, \theta)$ and $Z(\theta)=Z(a, \theta)$ denote either the boundary of each island or the shell centerline given in parametric form by the spectral representations; and $\theta, \theta^{\prime}$ indicate the field point and the source point, respectively. The surface current density $K_{T, s}(\theta)$ on the shell centerline is given by the Fourier series defined in Section 2. The equivalent toroidal surface current density on the flux surfaces inside each island is defined by

$$
K_{T}(\rho, \theta)=\frac{\widehat{n} \cdot \nabla \Phi_{P}}{2 \pi \mu_{0} h_{\zeta}(\rho, \theta)}=\frac{|\nabla \rho|}{2 \pi \mu_{0} h_{\zeta}(\rho, \theta)} \frac{d \Phi_{P}}{d \rho}=\frac{1}{\mu_{0}}\left(\frac{h_{\theta}(\rho, \theta)}{2 \pi \sqrt{g}(\rho, \theta)}\right) \frac{d \Phi_{P}}{d \rho},
$$

where $\rho, \theta, \zeta$ form a right-handed flux coordinates system, and $\Phi_{P}(\rho)$ is the poloidal flux contained by a given flux surface in the island (the total poloidal flux between the symmetry axis and the given flux surface is $\Phi=\Phi_{m}-\Phi_{P}$, where $\Phi_{m}$ is the value of $\Phi$ on the magnetic axis). The geometric factors in this formula, besides the previously defined poloidal scale factor $h_{\theta}(\rho, \theta)=\sqrt{(\partial R / \partial \theta)^{2}+(\partial Z / \partial \theta)^{2}}$, are the toroidal scale factor $h_{\zeta}(\rho, \theta)=R(\rho, \theta)$ (distance to the symmetry axis) and the Jacobian

$$
\sqrt{g}(\rho, \theta)=R(\rho, \theta)\left(\frac{\partial R}{\partial \rho} \frac{\partial Z}{\partial \theta}-\frac{\partial R}{\partial \theta} \frac{\partial Z}{\partial \rho}\right) .
$$

These quantities can be easily evaluated from the spectral representations and corresponding Fourier coefficients given in Sections 2 and 3. The relation between the poloidal flux function $\Phi_{P}$ and the total toroidal current $I_{T}$ contained by a flux surface is

$$
\frac{d \Phi_{P}}{d \rho}=\frac{I_{T}(\rho)}{K(\rho)},
$$

where

$$
K(\rho)=\frac{1}{2 \pi \mu_{0}} \oint_{\ell}\left(\frac{h_{\theta}(\rho, \theta)}{\sqrt{g}(\rho, \theta)}\right) d \ell(\theta)=\frac{1}{\mu_{0}}\left\langle\frac{h_{\theta}^{2}(\rho, \theta)}{\sqrt{g}(\rho, \theta)}\right\rangle_{\theta} .
$$

The magnetic coefficient $K(\rho)$ can be evaluated analytically by the method of residues [16]. But, the analytic results are convenient only in simple or limiting cases. In general, the poloidal-angle averages can be numerically calculated by Gauss-Chebyshev quadrature with adequate precision using few terms $(n>2 N$ where $N$ is the Fourier series order in the spectral representation; it may be advantageous to oversample such that $n \geq 4 N$ ):

$$
\langle f(\theta)\rangle_{\theta}=\frac{1}{2 \pi} \int_{0}^{2 \pi} f(\theta) d \theta \cong \frac{1}{2 n} \sum_{k=1}^{n}\left[f\left(\theta_{k}\right)+f\left(\pi+\theta_{k}\right)\right] .
$$

Here $\theta_{k}=(2 k-1) \pi /(2 n)$, which corresponds to an optimum integration mesh in the poloidal direction. For symmetric integrands this reduces to

$$
\left\langle f^{(s)}(\theta)\right\rangle_{\theta}=\frac{1}{\pi} \int_{0}^{\pi} f^{(s)}(\theta) d \theta \cong \frac{1}{n} \sum_{k=1}^{n} f^{(s)}\left(\theta_{k}\right) .
$$


Now, one can take symmetric

$$
\Phi_{i}\left(a_{i}\right) \delta_{n, 0}=L_{i i, n}^{(s)} I_{T, i}+M_{j i, n}^{(s)} I_{T, j}+M_{s i, 0 n}^{(s, s)} I_{T, s}+\sum_{m=1}^{n_{s}}\left(M_{s i, m n}^{(s, s)} I_{m}^{(s)}+M_{s i, m n}^{(a, s)} I_{m}^{(a)}\right)
$$

and antisymmetric

$$
0=L_{i i, n}^{(a)} I_{T, i}+M_{j i, n}^{(a)} I_{T, j}+M_{s i, 0 n}^{(s, a)} I_{T, s}+\sum_{m=1}^{n_{s}}\left(M_{s i, m n}^{(s, a)} I_{m}^{(s)}+M_{s i, m n}^{(a, a)} I_{m}^{(a)}\right)
$$

moments of the flux function $\Phi_{i}\left(a_{i}, \theta_{i}\right)=\Phi_{i}\left(a_{i}\right)=$ constant (Dirichlet condition) on the boundary of the island $i$ (note that the shell is assumed up-down symmetric but not the islands). The "self" and "mutual" inductance coefficients are defined by

$$
\begin{aligned}
L_{i i, n}^{(s)} & =\frac{1}{K_{i}\left(a_{i}\right)}\left\langle\left\langle\left(\frac{h_{\theta}^{2}\left(a_{i}, \theta_{i}^{\prime}\right)}{\sqrt{g}\left(a_{i}, \theta_{i}^{\prime}\right)}\right) G\left(\theta_{i} ; \theta_{i}^{\prime}\right)\right\rangle_{\theta_{i}^{\prime}} \cos n \theta_{i}\right\rangle_{\theta_{i}}, \\
M_{j i, n}^{(s)} & =\frac{1}{K_{j}\left(a_{j}\right)}\left\langle\left\langle\left(\frac{h_{\theta}^{2}\left(a_{j}, \theta_{j}\right)}{\sqrt{g}\left(a_{j}, \theta_{j}\right)}\right) G\left(\theta_{i} ; \theta_{j}\right)\right\rangle_{\theta_{j}} \cos n \theta_{i}\right\rangle_{\theta_{i}}, \\
M_{s i, m n}^{(s, s)} & =\mu_{0}\left\langle\left\langle G\left(\theta_{i} ; \theta_{s}\right) \cos m \theta_{s}\right\rangle_{\theta_{s}} \cos n \theta_{i}\right\rangle_{\theta_{i}}, \\
M_{s i, m n}^{(a, s)} & =\mu_{0}\left\langle\left\langle G\left(\theta_{i} ; \theta_{s}\right) \sin m \theta_{s}\right\rangle_{\theta_{s}} \cos n \theta_{i}\right\rangle_{\theta_{i}},
\end{aligned}
$$

and

$$
\begin{aligned}
L_{i i, n}^{(a)} & =\frac{1}{K_{i}\left(a_{i}\right)}\left\langle\left\langle\left(\frac{h_{\theta}^{2}\left(a_{i}, \theta_{i}^{\prime}\right)}{\sqrt{g}\left(a_{i}, \theta_{i}^{\prime}\right)}\right) G\left(\theta_{i} ; \theta_{i}^{\prime}\right)\right\rangle_{\theta_{i}^{\prime}} \sin n \theta_{i}\right\rangle_{\theta_{i}}, \\
M_{j i, n}^{(a)} & =\frac{1}{K_{j}\left(a_{j}\right)}\left\langle\left\langle\left(\frac{h_{\theta}^{2}\left(a_{j}, \theta_{j}\right)}{\sqrt{g}\left(a_{j}, \theta_{j}\right)}\right) G\left(\theta_{i} ; \theta_{j}\right)\right\rangle_{\theta_{j}} \sin n \theta_{i}\right\rangle_{\theta_{i}}, \\
M_{s i, m n}^{(s, a)} & =\mu_{0}\left\langle\left\langle G\left(\theta_{i} ; \theta_{s}\right) \cos m \theta_{s}\right\rangle_{\theta_{s}} \sin n \theta_{i}\right\rangle_{\theta_{i}}, \\
M_{s i, m n}^{(a, a)} & =\mu_{0}\left\langle\left\langle G\left(\theta_{i} ; \theta_{s}\right) \sin m \theta_{s}\right\rangle_{\theta_{s}} \sin n \theta_{i}\right\rangle_{\theta_{i}} .
\end{aligned}
$$

Similar coefficients can be defined for the poloidal flux function $\Phi_{j}\left(a_{j}\right)=$ constant on the boundary of the island $j$, with $I_{T, i}=I_{T, i}\left(a_{i}\right)$ and $I_{T, j}=I_{T, j}\left(a_{j}\right)$ denoting the total toroidal currents in the islands $i$ and $j$, respectively. Note that $L_{i i, 0}^{(s)}=L_{i, \text { ext }}$ gives exactly the external inductance of the island $i$ under the assumption of constant $\Phi_{i}\left(a_{i}\right)$. The coefficients $M_{j i, n}$ and $M_{s i, m n}$ multiplied by the corresponding currents give the $n$-th order moments of the fluxes produced on the island $i$ by the island $j$ and by the $m$-th Fourier component of the shell current, respectively. They do not correspond to the actual mutual inductance coefficients between islands and shell. Indeed, the mutual inductance coefficient between the islands $i$ and $j$ is given by

$$
\begin{aligned}
M_{j i}=M_{i j} & =\frac{\mu_{0}}{I_{T, i}\left(a_{i}\right) I_{T, j}\left(a_{j}\right)} \oint_{\ell_{i}} K_{T, i}\left(a_{i}, \theta_{i}\right) d \ell\left(\theta_{i}\right) \oint_{\ell_{j}} K_{T, j}\left(a_{j}, \theta_{j}\right) G\left(\theta_{i} ; \theta_{j}\right) d \ell\left(\theta_{j}\right) \\
& =\frac{1}{\mu_{0} K_{i}\left(a_{i}\right) K_{j}\left(a_{j}\right)}\left\langle\left(\frac{h_{\theta}^{2}\left(a_{i}, \theta_{i}\right)}{\sqrt{g}\left(a_{i}, \theta_{i}\right)}\right)\left\langle\left(\frac{h_{\theta}^{2}\left(a_{j}, \theta_{j}\right)}{\sqrt{g}\left(a_{j}, \theta_{j}\right)}\right) G\left(\theta_{i} ; \theta_{j}\right)\right\rangle_{\theta_{j}}\right\rangle_{\theta_{i}},
\end{aligned}
$$


and between the island $i$ and the shell $s$ by

$$
\begin{aligned}
M_{s i}=M_{i s}= & \frac{1}{K_{i}\left(a_{i}\right)}\left\langle\left(\frac{h_{\theta}^{2}\left(a_{i}, \theta_{i}\right)}{\sqrt{g}\left(a_{i}, \theta_{i}\right)}\right)\left\langle\left(\frac{2 \pi h_{\theta, s}\left(\theta_{s}\right) K_{T, s}\left(\theta_{s}\right)}{I_{T, s}}\right) G\left(\theta_{i} ; \theta_{s}\right)\right\rangle_{\theta_{s}}\right\rangle_{\theta_{i}} \\
= & \frac{1}{K_{i}\left(a_{i}\right)}\left\langle\left(\frac{h_{\theta}^{2}\left(a_{i}, \theta_{i}\right)}{\sqrt{g}\left(a_{i}, \theta_{i}\right)}\right)\left\langle G\left(\theta_{i} ; \theta_{s}\right)\right\rangle_{\theta_{s}}\right\rangle_{\theta_{i}} \\
& +\sum_{m=1}^{n_{s}} \frac{I_{m}^{(s)} / I_{T, s}}{K_{i}\left(a_{i}\right)}\left\langle\left(\frac{h_{\theta}^{2}\left(a_{i}, \theta_{i}\right)}{\sqrt{g}\left(a_{i}, \theta_{i}\right)}\right)\left\langle G\left(\theta_{i} ; \theta_{s}\right) \cos m \theta_{s}\right\rangle_{\theta_{s}}\right\rangle_{\theta_{i}} \\
& +\sum_{m=1}^{n_{s}} \frac{I_{m}^{(a)} / I_{T, s}}{K_{i}\left(a_{i}\right)}\left\langle\left(\frac{h_{\theta}^{2}\left(a_{i}, \theta_{i}\right)}{\sqrt{g}\left(a_{i}, \theta_{i}\right)}\right)\left\langle G\left(\theta_{i} ; \theta_{s}\right) \sin m \theta_{s}\right\rangle_{\theta_{s}}\right\rangle_{\theta_{i}} \\
= & \frac{1}{I_{T, s}}\left[M_{s i, 0}^{(s)} I_{T, s}+\sum_{m=1}^{n_{s}}\left(M_{s i, m}^{(s)} I_{m}^{(s)}+M_{s i, m}^{(a)} I_{m}^{(a)}\right)\right]
\end{aligned}
$$

The moment equations for the flux $\Phi_{i}\left(a_{i}\right)$ on the boundary of the island $i$ reflect the fact that all the external sources taken together, plus the current in the island $i$ itself, produce a constant poloidal flux on the boundary. This is not true, in general, for each source taken separately. However, for filamentary islands with $a_{i} \rightarrow 0, R_{0, i}\left(a_{i}\right) \rightarrow R_{m, i}, Z_{0, i}\left(a_{i}\right) \rightarrow$ $Z_{m, i}, e_{i}\left(a_{i}\right) \rightarrow \kappa_{m, i} \cong 1$ the previous expressions are greatly simplified, eliminating the dependency on the internal current distribution

$$
\frac{h_{\theta}^{2}\left(a_{i}, \theta_{i}\right)}{\sqrt{g}\left(a_{i}, \theta_{i}\right)} \underset{a_{i} \rightarrow 0}{\rightarrow} \frac{a_{i}}{R_{m, i}} \quad, \quad K_{i}\left(a_{i}\right) \underset{a_{i} \rightarrow 0}{\rightarrow} \frac{a_{i}}{\mu_{0} R_{m, i}},
$$

and

$$
\begin{aligned}
& L_{i i, 0}^{(s)}=L_{i, \mathrm{ext}} \underset{a_{i} \rightarrow 0}{\rightarrow} \mu_{0}\left\langle\left\langle G\left(\theta_{i} ; \theta_{i}^{\prime}\right)\right\rangle_{\theta_{i}^{\prime}}\right\rangle_{\theta_{i}}, \\
& M_{j i} \underset{a_{i}, a_{j} \rightarrow 0}{\rightarrow} \mu_{0}\left\langle\left\langle G\left(\theta_{i} ; \theta_{j}\right)\right\rangle_{\theta_{j}}\right\rangle_{\theta_{i}} \\
& M_{s i, m}^{(s)} \underset{a_{i} \rightarrow 0}{\rightarrow} \quad M_{s i, m 0}^{(s, s)}=\mu_{0}\left\langle\left\langle G\left(\theta_{i} ; \theta_{s}\right) \cos m \theta_{s}\right\rangle_{\theta_{s}}\right\rangle_{\theta_{i}}, \\
& M_{s i, m}^{(a)} \underset{a_{i} \rightarrow 0}{\rightarrow} M_{s i, m 0}^{(a, s)}=\mu_{0}\left\langle\left\langle G\left(\theta_{i} ; \theta_{s}\right) \sin m \theta_{s}\right\rangle_{\theta_{s}}\right\rangle_{\theta_{i}} .
\end{aligned}
$$

In the symmetric shell case (as is the present one) the moment equations for the flux function $\Phi_{s}\left(a_{s}, \theta_{s}\right)$ take the symmetric form

$$
\Phi_{s}\left(a_{s}\right) \delta_{n, 0}=L_{s s, 0 n}^{(s)} I_{T, s}+\sum_{m=1}^{n_{s}} L_{s s, m n}^{(s)} I_{m}^{(s)}+M_{i s, n}^{(s)} I_{T, i}+M_{j s, n}^{(s)} I_{T, j}
$$

and the antisymmetric one

$$
0=\sum_{m=1}^{n_{s}} L_{s s, m n}^{(a)} I_{m}^{(a)}+M_{i s, n}^{(a)} I_{T, i}+M_{j s, n}^{(a)} I_{T, j}
$$

where

$$
\begin{aligned}
L_{s s, m n}^{(s)} & =\mu_{0}\left\langle\left\langle G\left(\theta_{s} ; \theta_{s}^{\prime}\right) \cos m \theta_{s}^{\prime}\right\rangle_{\theta_{s}^{\prime}} \cos n \theta_{s}\right\rangle_{\theta_{s}}, \\
M_{i s, n}^{(s)} & =\frac{1}{K_{i}\left(a_{i}\right)}\left\langle\left\langle\left(\frac{h_{\theta}^{2}\left(a_{i}, \theta_{i}\right)}{\sqrt{g}\left(a_{i}, \theta_{i}\right)}\right) G\left(\theta_{s} ; \theta_{i}\right)\right\rangle_{\theta_{i}} \cos n \theta_{s}\right\rangle_{\theta_{s}}=M_{s i, n}^{(s)}
\end{aligned}
$$


and

$$
\begin{aligned}
L_{s s, m n}^{(a)} & =\mu_{0}\left\langle\left\langle G\left(\theta_{s} ; \theta_{s}^{\prime}\right) \sin m \theta_{s}^{\prime}\right\rangle_{\theta_{s}^{\prime}} \sin n \theta_{s}\right\rangle_{\theta_{s}}, \\
M_{i s, n}^{(a)} & =\frac{1}{K_{i}\left(a_{i}\right)}\left\langle\left\langle\left(\frac{h_{\theta}^{2}\left(a_{i}, \theta_{i}\right)}{\sqrt{g}\left(a_{i}, \theta_{i}\right)}\right) G\left(\theta_{s} ; \theta_{i}\right)\right\rangle_{\theta_{i}} \sin n \theta_{s}\right\rangle_{\theta_{s}}=M_{s i, n}^{(a)} .
\end{aligned}
$$

The "self-inductance" coefficients of the containing shell depend only of the shell shape. They have the following symmetry properties:

$$
L_{s s, m n}^{(s)}=L_{s s, n m}^{(s)} \quad, \quad L_{s s, m n}^{(a)}=L_{s s, n m}^{(a)} .
$$

Furthermore, because the Fourier components of the surface current density are weakly coupled, the self-linked flux is described by nearly band matrices.

The calculation of the "self-inductance" coefficients $L_{s s, m n}^{(s)}$ and $L_{s s, m n}^{(a)}$ requires some attention because of the singular character of the Green's function [19]:

$$
G\left(\theta ; \theta^{\prime}\right) \underset{\theta^{\prime} \rightarrow \theta}{\longrightarrow}-h_{\zeta}(\theta)\left\{\frac{1}{2} \ln \left[\left(\frac{h_{\theta}(\theta)}{8 h_{\zeta}(\theta)}\right) 2 \sin \left(\frac{\theta-\theta^{\prime}}{2}\right)\right]^{2}+2\right\} .
$$

Introducing the dimensionless auxiliary function

$$
g\left(\theta ; \theta^{\prime}\right)=\frac{G\left(\theta ; \theta^{\prime}\right)}{h_{\zeta}(\theta)}+\left\{\frac{1}{2} \ln \left[\left(\frac{h_{\theta}(\theta)}{8 h_{\zeta}(\theta)}\right) 2 \sin \left(\frac{\theta-\theta^{\prime}}{2}\right)\right]^{2}+2\right\} \underset{\theta^{\prime} \rightarrow \theta}{\longrightarrow} 0,
$$

which is nonsingular but nonsymmetric (not a true Green's function), the expressions for the "self-inductance" coefficients become

$$
\begin{aligned}
L_{m n}^{(s)}= & \mu_{0}\left\langle h_{\zeta}(\theta)\left\langle g\left(\theta ; \theta^{\prime}\right) \cos m \theta^{\prime}\right\rangle_{\theta^{\prime}} \cos n \theta\right\rangle_{\theta} \\
& -\mu_{0}\left\langle h_{\zeta}(\theta)\left\langle\left\{\frac{1}{2} \ln \left[\left(\frac{h_{\theta}(\theta)}{8 h_{\zeta}(\theta)}\right) 2 \sin \left(\frac{\theta-\theta^{\prime}}{2}\right)\right]^{2}+2\right\} \cos m \theta^{\prime}\right\rangle_{\theta^{\prime}} \cos n \theta\right\rangle_{\theta},
\end{aligned}
$$

and

$$
\begin{aligned}
L_{m n}^{(a)}= & \mu_{0}\left\langle h_{\zeta}(\theta)\left\langle g\left(\theta ; \theta^{\prime}\right) \sin m \theta^{\prime}\right\rangle_{\theta^{\prime}} \sin n \theta\right\rangle_{\theta} \\
& -\mu_{0}\left\langle h_{\zeta}(\theta)\left\langle\left\{\frac{1}{2} \ln \left[\left(\frac{h_{\theta}(\theta)}{8 h_{\zeta}(\theta)}\right) 2 \sin \left(\frac{\theta-\theta^{\prime}}{2}\right)\right]^{2}+2\right\} \sin m \theta^{\prime}\right\rangle_{\theta^{\prime}} \sin n \theta\right\rangle_{\theta} .
\end{aligned}
$$

Using the integrals

$$
\begin{aligned}
& \frac{1}{4 \pi} \int_{0}^{2 \pi} \ln \left[2 \sin \left(\frac{\theta-\theta^{\prime}}{2}\right)\right]^{2} \cos m \theta^{\prime} d \theta^{\prime}=-\frac{\cos m \theta}{2 m}\left(1-\delta_{m, 0}\right), \\
& \frac{1}{4 \pi} \int_{0}^{2 \pi} \ln \left[2 \sin \left(\frac{\theta-\theta^{\prime}}{2}\right)\right]^{2} \sin m \theta^{\prime} d \theta^{\prime}=-\frac{\sin m \theta}{2 m}\left(1-\delta_{m, 0}\right),
\end{aligned}
$$

it follows that

$$
\begin{aligned}
L_{s s, m n}^{(s)}= & \mu_{0}\left\langle h_{\zeta, s}(\theta)\left\langle g_{s}\left(\theta ; \theta^{\prime}\right) \cos m \theta^{\prime}\right\rangle_{\theta^{\prime}} \cos n \theta\right\rangle_{\theta} \\
& +\mu_{0} \delta_{m, 0}\left\langle h_{\zeta, s}(\theta)\left[\ln \left(\frac{8 h_{\zeta, s}(\theta)}{h_{\theta, s}(\theta)}\right)-2\right] \cos n \theta\right\rangle_{\theta} \\
& +\mu_{0}\left(\frac{1-\delta_{m, 0}}{2 m}\right)\left\langle h_{\zeta, s}(\theta) \cos m \theta \cos n \theta\right\rangle_{\theta},
\end{aligned}
$$


and

$$
\begin{aligned}
L_{s s, m n}^{(a)}= & \mu_{0}\left\langle h_{\zeta, s}(\theta)\left\langle g_{s}\left(\theta ; \theta^{\prime}\right) \sin m \theta^{\prime}\right\rangle_{\theta^{\prime}} \sin n \theta\right\rangle_{\theta} \\
& +\mu_{0}\left(\frac{1-\delta_{m, 0}}{2 m}\right)\left\langle h_{\zeta, s}(\theta) \sin m \theta \sin n \theta\right\rangle_{\theta} .
\end{aligned}
$$

The logarithmic term in $L_{s s, 0 n}^{(s)}$ corresponds to the self-field contribution to the inductance of the shell. Likewise, the coefficients $L_{i i, n}^{(s)}$ and $L_{i i, n}^{(a)}$ (or $L_{j j, n}^{(s)}$ and $L_{j j, n}^{(a)}$ ) can be written as

$$
\begin{aligned}
L_{i i, n}^{(s)}= & \frac{1}{K_{i}\left(a_{i}\right)}\left\langle\left(\frac{h_{\theta}^{2}\left(a_{i}, \theta\right) h_{\zeta}\left(a_{i}, \theta\right)}{\sqrt{g}\left(a_{i}, \theta\right)}\right)\left(\left\langle g_{i}\left(\theta ; \theta^{\prime}\right) \cos n \theta^{\prime}\right\rangle_{\theta^{\prime}}+\frac{\cos n \theta}{2 n}\left(1-\delta_{n, 0}\right)\right)\right\rangle_{\theta} \\
& +\frac{\delta_{n, 0}}{K_{i}\left(a_{i}\right)}\left\langle\left(\frac{h_{\theta}^{2}\left(a_{i}, \theta\right) h_{\zeta}\left(a_{i}, \theta\right)}{\sqrt{g}\left(a_{i}, \theta\right)}\right)\left[\ln \left(\frac{8 h_{\zeta}\left(a_{i}, \theta\right)}{h_{\theta}\left(a_{i}, \theta\right)}\right)-2\right]\right\rangle_{\theta}, \\
L_{i i, n}^{(a)}= & \frac{1}{K_{i}\left(a_{i}\right)}\left\langle\left(\frac{h_{\theta}^{2}\left(a_{i}, \theta\right) h_{\zeta}\left(a_{i}, \theta\right)}{\sqrt{g}\left(a_{i}, \theta\right)}\right)\left(\left\langle g_{i}\left(\theta ; \theta^{\prime}\right) \sin n \theta^{\prime}\right\rangle_{\theta^{\prime}}+\frac{\sin n \theta}{2 n}\left(1-\delta_{n, 0}\right)\right)\right\rangle_{\theta} .
\end{aligned}
$$

Now, for ideal equilibrium the current distribution $K_{T, s}(\theta, t=0)$ in the containing shell adjusts itself to give a constant value of the flux $\Phi_{s}(t=0)$ on the shell. In the same way, the shapes of the magnetic islands adjust themselves to give constant flux values $\Phi_{i}\left(\rho=a_{i}, t=0\right)$ and $\Phi_{j}\left(\rho=a_{j}, t=0\right)$ at the plasma edges. The values of the fluxes depend of the geometries of both islands and shell and of the total (given) values of the toroidal currents. In other words, one has a free-boundary problem with Dirichlet conditions for both islands that has to be solved simultaneously with the current distribution induced in the shell. In general, the shape of the containing shell is determined by the external sources, but it is assumed fixed in the present problem. In this case, the current distribution in the shell provides Neumann conditions for the external equilibrium problem which is solved in Section 5. Using the method of moments, the Dirichlet problem is reduced to a root finding procedure, involving a set of coupled equations which is nonlinear in the islands parameters and linear in the shell current (Fourier) components, namely equations 21, 22 for the islands $i$ and $j$, and equations 29 and 30 for the shell $s$. The internal equilibrium of each island is determined simultaneously solving equations 9 , 10 and 11, which depend of the pressure and toroidal current profiles inside the islands. In this paper these profiles take the following simple polynomial forms in $\rho$ :

$$
\begin{aligned}
p(\rho) & =p(0)\left(\frac{a^{2}-\rho^{2}}{a^{2}}\right)^{2}\left[1+\left(2+\frac{a^{2} p^{\prime \prime}(0)}{2 p(0)}\right) \frac{\rho^{2}}{a^{2}}\right], \quad-6<\frac{a^{2} p^{\prime \prime}(0)}{p(0)}<0, \\
I_{T}(\rho) & =I_{T}(a)\left(1-\frac{\rho^{2}}{2 a^{2}}\right) \frac{2 \rho^{2}}{a^{2}} .
\end{aligned}
$$

For further simplicity it is assumed that $p^{\prime \prime}(0)=-6 p(0) / a^{2}$, which is the minimum value giving peaked profiles and positive pressure in the whole radial range $0 \leq \rho \leq a$ $\left(p^{\prime \prime}(0)<0\right.$ for non-hollow profiles). The pressure on the island magnetic axis is

$$
p_{0}=p(0)=\left(10^{3} e\right) n_{e}(0)\left(T_{e}(0)+\frac{T_{i}(0)}{Z_{\mathrm{eff}}}\right)
$$

with $T_{e}(0)$ and $T_{i}(0)$ in $\mathrm{keV}$ ( $e$ is the electron charge). The following plasma parameters are assumed for both islands: $n_{e}(0)=0.5 \times 10^{20} \mathrm{~m}^{-3}, T_{e}(0)=T_{i}(0)=1 \mathrm{keV}, Z_{\text {eff }}=1.5$. The values of the plasma current in the islands are [17]: $I_{T, i}(0)=-3.92 \mathrm{MA}$ (inner island in the figures), $I_{T, j}(0)=4.52 \mathrm{MA}$ (outer island). The total current in the containing 


\begin{tabular}{|l|l||l|l|}
\hline \multicolumn{2}{|l||}{ Island $i$} & \multicolumn{2}{l|}{ Island $j$} \\
\hline \hline$R_{0, i}(\mathrm{~m})$ & 2.953 & $R_{0, j}(\mathrm{~m})$ & 3.749 \\
\hline$a_{i}(\mathrm{~m})$ & 0.263 & $a_{j}(\mathrm{~m})$ & 0.381 \\
\hline$e_{i}$ & 1.693 & $e_{j}$ & 1.255 \\
\hline$S_{2, i}$ & -0.0714 & $S_{2, j}$ & 0.0582 \\
\hline$S_{3, i}$ & -0.0134 & $S_{3, j}$ & -0.0166 \\
\hline$R_{m, i}(\mathrm{~m})$ & 2.966 & $R_{m, j}(\mathrm{~m})$ & 3.738 \\
\hline$\kappa_{m, i}$ & 1.558 & $\kappa_{m, j}$ & 1.215 \\
\hline$p_{0, i}(\mathrm{kPa})$ & 13.35 & $p_{0, j}(\mathrm{kPa})$ & 13.35 \\
\hline$\Phi_{i}(\mathrm{~Wb})$ & 5.330 & $\Phi_{j}(\mathrm{~Wb})$ & 14.180 \\
\hline
\end{tabular}

Table 1: Parameters of the islands for the symmetric equilibrium shown in Fig. 1. The values of the Fourier coefficients correspond to the edge values.

shell is $I_{T, s}(0)=I_{T, i}(0)+I_{T, j}(0)=600 \mathrm{kA}$, that is, one neglects the possible current contribution in the region between the islands and the containing shell.

The equilibrium depends of the initial conditions in the islands. Therefore, in order to solve the equilibrium it is necessary to specify two geometrical parameters (one for each island). In a tokamak equilibrium problem this corresponds to specifying the limiter or $\mathrm{X}$-point positions. In the present problem it is convenient to fix the initial values of the minor radii, which set the scale of the islands, or, equivalently, the gaps between islands and containing shell. Then, the values of the total poloidal fluxes $\Phi_{s}(t=0)=\Phi_{s}(0)$, $\Phi_{i}\left(\rho=a_{i}, t=0\right)=\Phi_{i}(0)$ and $\Phi_{j}\left(\rho=a_{j}, t=0\right)=\Phi_{j}(0)$ are determined from the zerothorder moment equations $(n=0)$. The shapes of the islands and the current distribution in the shell evolve with time from this initial equilibrium. Thus in Section 6 a small tilting displacement is analyzed with fixed values of the total toroidal currents and fluxes contained by the islands, corresponding to the initial equilibrium values, though other parameters may vary completely. One must point out that the root-finding procedure depends of good starting values. It may be necessary to start the problem with less independent variables and gradually introduce higher-order corrections in the shape of the islands involving triangularity, quadrangularity etc.

Table 1 gives the equilibrium parameters calculated for the symmetric configuration shown in Fig. 1, i.e., an equilibrium that is symmetric with respect to the plane $Z=0$ with $A_{i, n}=0, A_{j, n}=0$ and $I_{m}^{(a)}=0$. The surface current calculation in the containing shell is limited to four Fourier coefficients $\left(n_{s}=4\right)$ which take the following values in equilibrium: $I_{1}^{(s)}(0)=-8.198 \mathrm{MA}, I_{2}^{(s)}(0)=2.549 \mathrm{MA}, I_{3}^{(s)}(0)=312.7 \mathrm{kA}, I_{4}^{(s)}(0)=-169.3 \mathrm{kA}$. The surface current and the self-flux distributions in the containing shell are shown in Fig. 2. Addition of this self-flux to the poloidal flux produced by the two islands results in a constant flux value $\Phi_{s}(0)=7.830 \mathrm{~Wb}$ on the shell. The poloidal flux values between the symmetry axis and the islands contours are $\Phi_{i}(0)=5.330 \mathrm{~Wb}$ and $\Phi_{j}(0)=14.180 \mathrm{~Wb}$. The poloidal fluxes contained by the islands, that is, between the magnetic axes and the islands contours are $\Phi_{P, i}(0)=-10.010 \mathrm{~Wb}$ and $\Phi_{P, j}(0)=15.750 \mathrm{~Wb}$. These flux values correspond to gaps $\delta\left(a_{i}\right)=\left(R_{0}\left(a_{i}\right)-a_{i}\right)-\left(R_{0, s}-a_{s}\right)=60 \mathrm{~mm}$ and $\delta\left(a_{j}\right)=$ $\left(R_{0, s}+a_{s}\right)-\left(R_{0}\left(a_{j}\right)+a_{j}\right)=140 \mathrm{~mm}$ measured along the equatorial plane between the boundaries of the inner and outer island, respectively, and the containing shell. The gaps were fixed initially to set the scale of the islands.

Figures 3 and 4 show the toroidal and poloidal current densities along the equatorial plane inside the magnetic islands. In general, the toroidal current density in the islands 


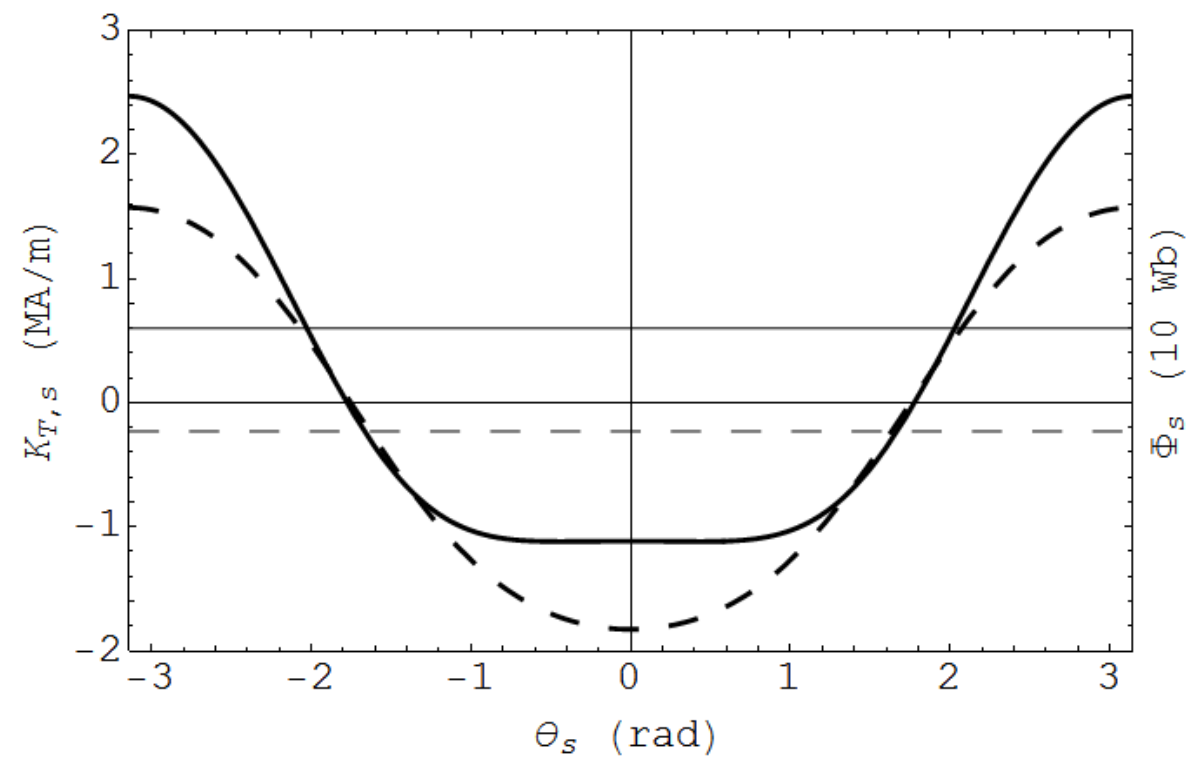

Figure 2: Surface current distribution (thick continuous line) and self-flux distribution (thick dashed line) in the containing shell for the symmetric equilibrium shown in Fig. 1. The thin continuous line corresponds to the total toroidal current flowing in the shell, $I_{T, s}=2 \pi\left\langle h_{\theta, s}(\theta) K_{T, s}(\theta, t)\right\rangle_{\theta}=600 \mathrm{kA}$, and the thin dashed line to the poloidal-angle averaged self-flux in the shell centerline, $\left\langle\Phi_{s}\right\rangle_{\theta}=-2.316 \mathrm{~Wb}$.

is given by

$$
j_{T}(\rho, \theta)=\frac{\mu_{0} d I_{T} / d \rho}{2 \pi h_{\zeta}(\rho, \theta) d L / d \rho}-\frac{K(\rho)}{I_{T}(\rho)}\left(2 \pi h_{\zeta}(\rho, \theta)-\frac{\mu_{0} d V / d \rho}{2 \pi h_{\zeta}(\rho, \theta) d L / d \rho}\right) \frac{d p}{d \rho},
$$

where $V(\rho)$ is the volume enclosed by a flux surface

$$
\begin{aligned}
V(\rho) & =\pi \int_{0}^{2 \pi}\left(R^{2}(\rho, \theta) \frac{\partial Z}{\partial \theta}\right) d \theta \\
\frac{d V}{d \rho} & =4 \pi^{2}\langle\sqrt{g}(\rho, \theta)\rangle_{\theta}
\end{aligned}
$$

and $L(\rho)$ is the inductance of the toroidal solenoid defined by the flux surface

$$
\begin{aligned}
L(\rho) & =-\frac{\mu_{0}}{2 \pi} \int_{0}^{2 \pi}\left(\frac{Z(\rho, \theta)}{R(\rho, \theta)} \frac{\partial R}{\partial \theta}\right) d \theta, \\
\frac{d L}{d \rho} & =\mu_{0}\left\langle\frac{\sqrt{g}(\rho, \theta)}{h_{\zeta}^{2}(\rho, \theta)}\right\rangle .
\end{aligned}
$$

The poloidal current density is given in terms of the total poloidal current $I(\rho)$ by

$$
j_{P}(\rho, \theta)=-\frac{h_{\theta}(\rho, \theta)}{2 \pi \sqrt{g}(\rho, \theta)} \frac{d I}{d \rho}=\frac{h_{\theta}(\rho, \theta)}{2 \pi \sqrt{g}(\rho, \theta)} \frac{d I_{P}}{d \rho} .
$$




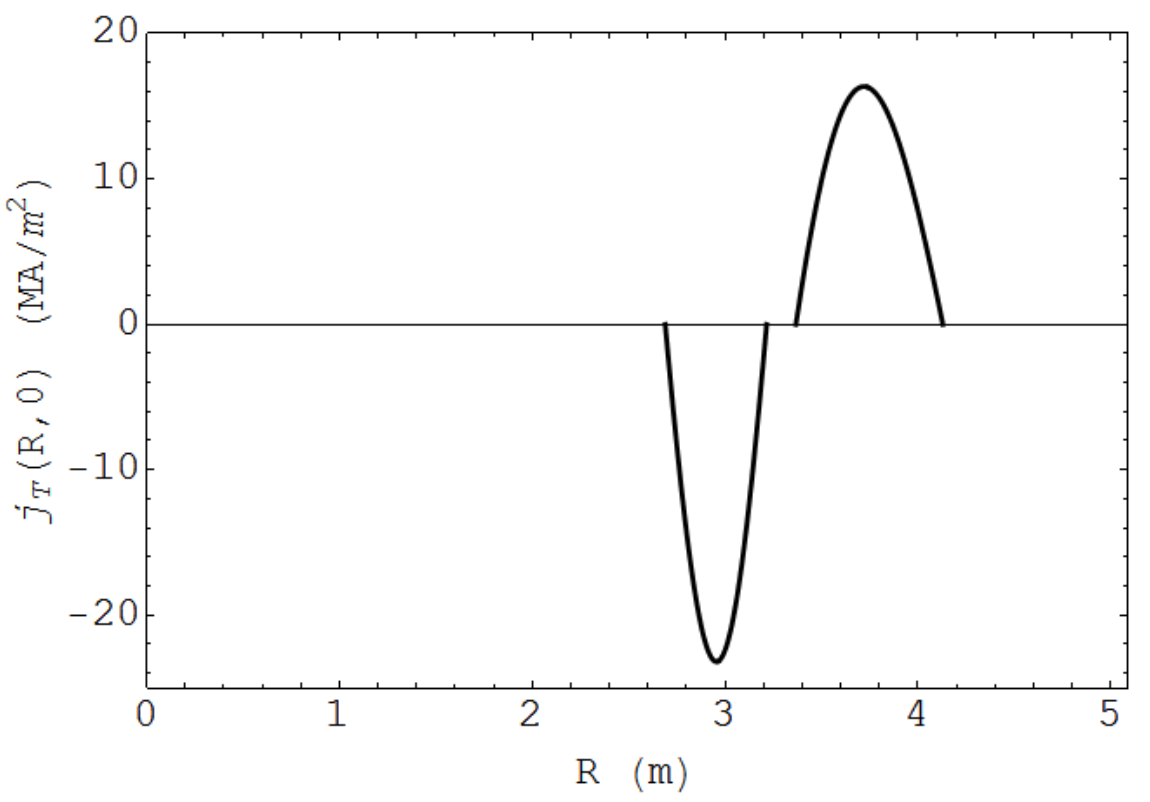

Figure 3: Toroidal current density profile along the equatorial plane inside the magnetic islands for the symmetric equilibrium shown in Fig. 1.

The total poloidal current $I(\rho)=I(0)-I_{P}(\rho)$ is evaluated in terms of the profiles of the total toroidal current $I_{T}(\rho)$ and of the pressure $p(\rho)$ by integration of the flux-surface averaged equilibrium equation

$$
\frac{d I^{2}}{d \rho}=-\frac{2}{d L / d \rho}\left(\frac{I_{T}(\rho)}{K(\rho)} \frac{d I_{T}}{d \rho}+\frac{d V}{d \rho} \frac{d p}{d \rho}\right)
$$

with the end-point condition $I(a)=2 \pi R_{0} B_{0} / \mu_{0}$, where $B_{0}$ is the external toroidal magnetic field at the major radius $R_{0}\left(R_{0}=3.4 \mathrm{~m}\right.$ and $B_{0}=3.7 \mathrm{~T}$ for JT-60U). The value $I(0)=2 \pi R_{m} B_{m} / \mu_{0}$ at the origin gives the magnetic induction $B_{m}$ on the magnetic axis of each island: $I_{i}(0)=86.87 \mathrm{MA}, B_{m, i}=5.86 \mathrm{~T} ; I_{j}(0)=95.25 \mathrm{MA}, B_{m, j}=5.10 \mathrm{~T}$. Hence, both islands are in strong paramagnetic equilibrium.

\section{$5 \quad$ External equilibrium magnetic field}

The total poloidal flux produced just outside the containing shell by the external sources is given by

$$
\Phi_{\mathrm{ext}}^{(+)}\left(\theta_{s}\right)=\Phi_{s}\left(a_{s}\right)-\mu_{0} \oint_{\ell_{s}^{(+)}} K_{T, s}\left(\theta_{s}^{\prime}\right) G\left(\theta_{s} ; \theta_{s}^{\prime}\right) d \ell\left(\theta_{s}^{\prime}\right),
$$

where $\Phi_{s}\left(a_{s}\right)$ is the constant flux on the shell and $K_{T, s}$ is the toroidal surface current density flowing in the shell contour $\ell_{s}$. In an axisymmetric system the component of the magnetic field tangential to a flux surface (Neumann boundary condition) is calculated in terms of the poloidal flux $\Phi$ by

$$
B_{\tau}=h_{\zeta}(\widehat{n} \times \vec{B}) \cdot \nabla \zeta=h_{\zeta}(\vec{B} \times \nabla \zeta) \cdot \widehat{n}=-\frac{\widehat{n} \cdot \nabla \Phi}{2 \pi h_{\zeta}},
$$

where $\widehat{n}$ is the unit vector pointing outwards normal to the flux surface. Accordingly, the tangential component of the magnetic field just outside the containing shell, due to 


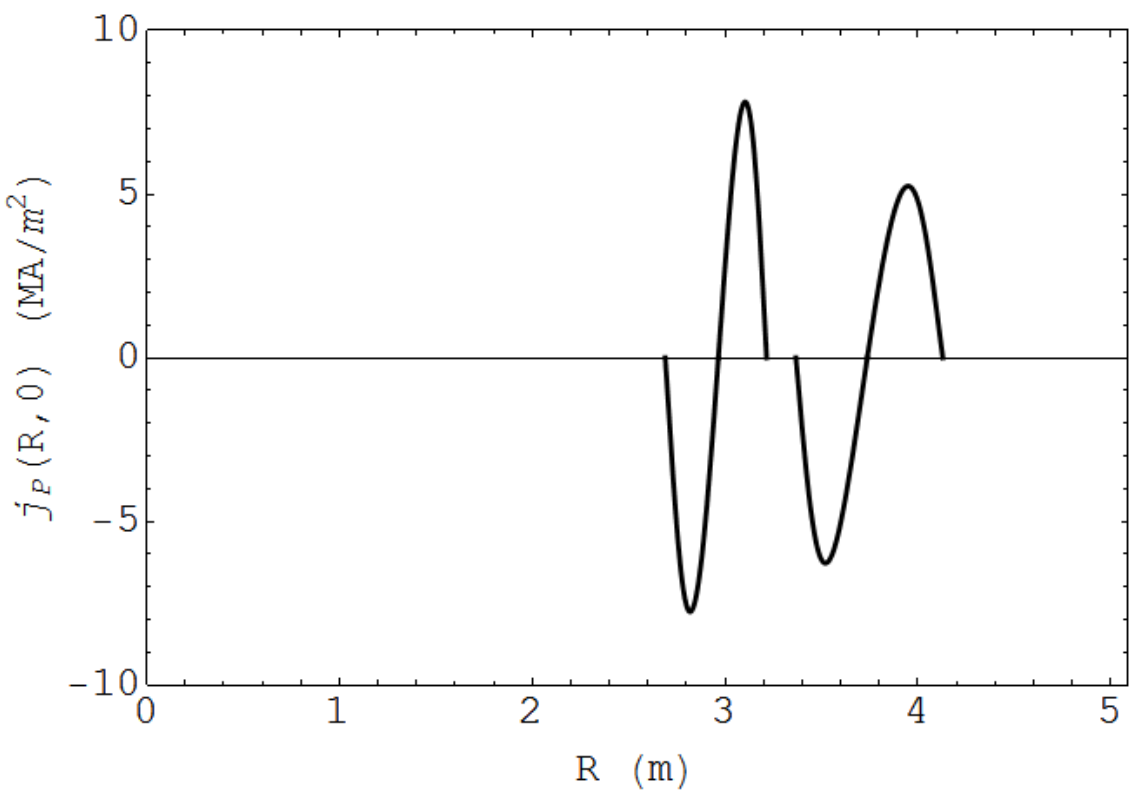

Figure 4: Poloidal current density profile along the equatorial plane inside the magnetic islands for the symmetric equilibrium shown in Fig. 1.

external sources, is

$$
\widehat{n} \times \vec{B}_{\text {ext }}^{(+)}\left(\theta_{s}\right)=h_{\zeta, s}(\theta) B_{\tau, \text { ext }}^{(+)}\left(\theta_{s}\right) \nabla \zeta=\left(\frac{\mu_{0}}{2 \pi} \oint_{\ell_{s}^{(+)}} K_{T, s}\left(\theta_{s}^{\prime}\right) \widehat{n} \cdot \nabla G\left(\theta_{s} ; \theta_{s}^{\prime}\right) d \ell\left(\theta_{s}^{\prime}\right)\right) \nabla \zeta .
$$

The normal component of $\vec{B}$ is continuous across a surface layer of current $\vec{K}=h_{\zeta} K_{T} \nabla \zeta$ but the tangential component experiences a discontinuity defined by

$$
\widehat{n} \times\left(\vec{B}_{(+)}-\vec{B}_{(-)}\right)=\mu_{0} \vec{K}
$$

Therefore, the tangential component of the magnetic field due to external sources is given just inside the containing shell by

$$
B_{\tau, \text { ext }}^{(-)}\left(\theta_{s}\right)=-\mu_{0} K_{T, s}\left(\theta_{s}\right)+\frac{\mu_{0}}{2 \pi h_{\zeta, s}\left(\theta_{s}\right)} \oint_{\ell_{s}^{(+)}} K_{T, s}\left(\theta_{s}^{\prime}\right) \widehat{n} \cdot \nabla G\left(\theta_{s} ; \theta_{s}^{\prime}\right) d \ell\left(\theta_{s}^{\prime}\right) .
$$

This expression corresponds to the maintaining field $\vec{B}^{(m)}=h_{\zeta}(\theta) B_{\tau, \text { ext }}^{(-)}(\theta) \nabla \zeta[20]$. Integration over the shell contour yields

$$
\begin{aligned}
\oint_{\ell_{s}} B_{\tau, \text { ext }}^{(-)}\left(\theta_{s}\right) d \ell\left(\theta_{s}\right)= & -\mu_{0} \oint_{\ell_{s}} K_{T, s}\left(\theta_{s}\right) d \ell\left(\theta_{s}\right) \\
& +\mu_{0} \oint_{\ell_{s}^{(+)}} K_{T, s}\left(\theta_{s}^{\prime}\right)\left(\oint_{\ell_{s}} \frac{\widehat{n} \cdot \nabla G\left(\theta_{s} ; \theta_{s}^{\prime}\right)}{2 \pi h_{\zeta, s}\left(\theta_{s}\right)} d \ell\left(\theta_{s}\right)\right) d \ell\left(\theta_{s}^{\prime}\right)
\end{aligned}
$$

where

$$
\oint_{\ell_{s}} \frac{\widehat{n} \cdot \nabla G\left(\theta_{s} ; \theta_{s}^{\prime}\right)}{2 \pi h_{\zeta, s}\left(\theta_{s}\right)} d \ell\left(\theta_{s}\right)=\left\{\begin{array}{cl}
1 & \text { inside the shell } \\
1 / 2 & \text { on the shell } \\
0 & \text { outside the shell }
\end{array}\right.
$$






Figure 5: Maintaining magnetic field for the symmetric equilibrium shown in Fig. 1. The zero-crossing points correspond to the X-point positions on the containing shell.

in accordance with Gauss's theorem and Ampère's law. For integration exactly on the shell centerline the expression of the tangential component of the external magnetic field (maintaining field) can be written as

$$
B_{\tau, \mathrm{ext}}\left(\theta_{s}\right)=-\frac{\mu_{0} K_{T, s}\left(\theta_{s}\right)}{2}+\frac{\mu_{0}}{2 \pi h_{\zeta, s}\left(\theta_{s}\right)} \oint_{\ell_{s}} K_{T, s}\left(\theta_{s}^{\prime}\right) \widehat{n} \cdot \nabla G\left(\theta_{s} ; \theta_{s}^{\prime}\right) d \ell\left(\theta_{s}^{\prime}\right) .
$$

Figure 5 shows the maintaining magnetic field for the symmetric equilibrium shown in Fig. 1. An approximation of the maintaining field can be obtained representing the external sources by a finite set of coils. The poloidal flux produced in the position $\vec{r}$ by a circular coil with total current $I_{k}$ at a fixed position $\vec{r}_{k}$ is

$$
\Phi_{k}(\vec{r})=\mu_{0} I_{k} G\left(\vec{r} ; \vec{r}_{k}\right) .
$$

Each coil produces a magnetic field component tangential to $\ell_{s}$ given by

$$
B_{\tau, k}(\theta)=-\frac{\widehat{n} \cdot \nabla \Phi_{k}}{2 \pi h_{\zeta}(\theta)}=-\frac{\mu_{0} I_{k}}{2 \pi h_{\zeta}(\theta)} \widehat{n} \cdot \nabla G\left(\theta ; \vec{r}_{k}\right)
$$

so that the currents in the coils can be determined by a least-squares technique in order to satisfy the approximate equation (strictly an ill-posed problem)

$$
\sum_{k} I_{k} \widehat{n} \cdot \nabla G\left(\theta_{s} ; \vec{r}_{k}\right) \cong \pi h_{\zeta, s}\left(\theta_{s}\right) K_{T, s}\left(\theta_{s}\right)-\oint_{\ell_{s}} K_{T, s}\left(\theta_{s}^{\prime}\right) \widehat{n} \cdot \nabla G\left(\theta_{s} ; \theta_{s}^{\prime}\right) d \ell\left(\theta_{s}^{\prime}\right) .
$$

Hence, the total poloidal flux of the vacuum field outside the shell is given by the sum of the flux produced by $K_{T, s}$ flowing on the shell centerline $\ell_{s}$ and the flux produced by the external coils:

$$
\Phi(\vec{r})=\mu_{0} \oint_{\ell_{s}} K_{T, s}\left(\theta_{s}^{\prime}\right) G\left(\vec{r} ; \theta_{s}^{\prime}\right) d \ell\left(\theta_{s}^{\prime}\right)+\mu_{0} \sum_{k} I_{k} G\left(\vec{r} ; \vec{r}_{k}\right) .
$$


The external equilibrium problem can be reduced to a small system of linear equations without strong oscillations by taking moments of the equilibrium equation. In this way, introducing the definitions

$$
\begin{aligned}
& N_{k, n}^{(s)}=\left\langle\widehat{n} \cdot \nabla G\left(\theta_{s} ; \vec{r}_{k}\right) \cos n \theta_{s}\right\rangle_{\theta_{s}}, \\
& N_{k, n}^{(a)}=\left\langle\widehat{n} \cdot \nabla G\left(\theta_{s} ; \vec{r}_{k}\right) \sin n \theta_{s}\right\rangle_{\theta_{s}}
\end{aligned}
$$

one obtains a system of symmetric and antisymmetric moment equations for the coils currents:

$$
\begin{aligned}
\sum_{k} N_{k, n}^{(s)} I_{k}= & \pi\left\langle h_{\zeta, s}\left(\theta_{s}\right) K_{T, s}\left(\theta_{s}\right) \cos n \theta_{s}\right\rangle_{\theta_{s}} \\
& -2 \pi\left\langle\left\langle h_{\theta, s}\left(\theta_{s}^{\prime}\right) K_{T, s}\left(\theta_{s}^{\prime}\right) \widehat{n} \cdot \nabla G\left(\theta_{s} ; \theta_{s}^{\prime}\right)\right\rangle_{\theta_{s}^{\prime}} \cos n \theta_{s}\right\rangle_{\theta_{s}}, \\
\sum_{k} N_{k, n}^{(a)} I_{k}= & \pi\left\langle h_{\zeta, s}\left(\theta_{s}\right) K_{T, s}\left(\theta_{s}\right) \sin n \theta_{s}\right\rangle_{\theta_{s}} \\
& -2 \pi\left\langle\left\langle h_{\theta, s}\left(\theta_{s}^{\prime}\right) K_{T, s}\left(\theta_{s}^{\prime}\right) \widehat{n} \cdot \nabla G\left(\theta_{s} ; \theta_{s}^{\prime}\right)\right\rangle_{\theta_{s}^{\prime}} \sin n \theta_{s}\right\rangle_{\theta_{s}}
\end{aligned}
$$

Defining the new coefficients

$$
\begin{aligned}
N_{m n}^{(s, s)} & =\left\langle\left(\frac{h_{\zeta, s}\left(\theta_{s}\right)}{2 h_{\theta, s}\left(\theta_{s}\right)} \cos m \theta_{s}-\left\langle\widehat{n} \cdot \nabla G\left(\theta_{s} ; \theta_{s}^{\prime}\right) \cos m \theta_{s}^{\prime}\right\rangle_{\theta_{s}^{\prime}}\right) \cos n \theta_{s}\right\rangle_{\theta_{s}}, \\
N_{m n}^{(s, a)} & =\left\langle\left(\frac{h_{\zeta, s}\left(\theta_{s}\right)}{2 h_{\theta, s}\left(\theta_{s}\right)} \sin m \theta_{s}-\left\langle\widehat{n} \cdot \nabla G\left(\theta_{s} ; \theta_{s}^{\prime}\right) \sin m \theta_{s}^{\prime}\right\rangle_{\theta_{s}^{\prime}}\right) \cos n \theta_{s}\right\rangle_{\theta_{s}}, \\
N_{m n}^{(a, s)} & =\left\langle\left(\frac{h_{\zeta, s}\left(\theta_{s}\right)}{2 h_{\theta, s}\left(\theta_{s}\right)} \cos m \theta_{s}-\left\langle\widehat{n} \cdot \nabla G\left(\theta_{s} ; \theta_{s}^{\prime}\right) \cos m \theta_{s}^{\prime}\right\rangle_{\theta_{s}^{\prime}}\right) \sin n \theta_{s}\right\rangle_{\theta_{s}}, \\
N_{m n}^{(a, a)} & =\left\langle\left(\frac{h_{\zeta, s}\left(\theta_{s}\right)}{2 h_{\theta, s}\left(\theta_{s}\right)} \sin m \theta_{s}-\left\langle\widehat{n} \cdot \nabla G\left(\theta_{s} ; \theta_{s}^{\prime}\right) \sin m \theta_{s}^{\prime}\right\rangle_{\theta_{s}^{\prime}}\right) \sin n \theta_{s}\right\rangle_{\theta_{s}},
\end{aligned}
$$

the system of equations (Neumann boundary conditions) that relate the currents in the equilibrium coils with the Fourier components of the toroidal surface current density in the shell becomes

$$
\begin{aligned}
\sum_{k} N_{k, n}^{(s)} I_{k} & =N_{0 n}^{(s, s)} I_{T, s}+\sum_{m=1}^{n_{s}}\left(N_{m n}^{(s, s)} I_{m}^{(s)}+N_{m n}^{(s, a)} I_{m}^{(a)}\right) \\
\sum_{k} N_{k, n}^{(a)} I_{k} & =N_{0 n}^{(a, s)} I_{T, s}+\sum_{m=1}^{n_{s}}\left(N_{m n}^{(a, s)} I_{m}^{(s)}+N_{m n}^{(a, a)} I_{m}^{(a)}\right) .
\end{aligned}
$$

It is possible to satisfy as many moment equations as the number of pairs of symmetric coils minus two, that is, for $n=0,1,2, \ldots k-2$, or to solve the equations using a leastsquares fitting procedure. A single pair of symmetric coils $(k=2)$ connected in series corresponds to a simple "vertical" equilibrium field.

Again it is necessary to take into account the singularity of the Green's function in numerical calculations. In general, the normal component of $\nabla G$ is given by

$$
\widehat{n} \cdot \nabla G\left(\theta ; \theta^{\prime}\right)=\frac{\operatorname{sign}\left[(\partial R / \partial \theta) \partial^{2} Z / \partial \theta^{2}-(\partial Z / \partial \theta) \partial^{2} R / \partial \theta^{2}\right]}{\sqrt{(\partial R / \partial \theta)^{2}+(\partial Z / \partial \theta)^{2}}}\left(-\frac{\partial Z}{\partial \theta} \frac{\partial G}{\partial R}+\frac{\partial R}{\partial \theta} \frac{\partial G}{\partial Z}\right)
$$


where

$$
\left\{\begin{aligned}
\frac{\partial G\left(R, Z ; R^{\prime}, Z^{\prime}\right)}{\partial R} & =\frac{\sqrt{m}}{2}\left\{K(m)-\left[1-\left(1+\frac{R^{\prime}}{R}\right) \frac{m}{2}\right] \frac{E(m)}{1-m}\right\} \sqrt{\frac{R}{R^{\prime}}} \\
\frac{\partial G\left(R, Z ; R^{\prime}, Z^{\prime}\right)}{\partial Z} & =\frac{\sqrt{m}}{2}\left[K(m)-\left(1-\frac{m}{2}\right) \frac{E(m)}{1-m}\right] \frac{Z-Z^{\prime}}{\sqrt{R R^{\prime}}} . \\
m & =\frac{4 R R^{\prime}}{\left(R+R^{\prime}\right)^{2}+\left(Z-Z^{\prime}\right)^{2}} \quad(0 \leqslant m \leqslant 1)
\end{aligned}\right.
$$

The "self-field" limit value of $\widehat{n} \cdot \nabla G$ is

$$
\begin{aligned}
\lim _{\theta^{\prime} \rightarrow \theta}\left[\widehat{n} \cdot \nabla G\left(\theta ; \theta^{\prime}\right)\right]= & \frac{\sqrt{h_{\theta}^{2}(\theta)-\left(\partial h_{\zeta} / \partial \theta\right)^{2}}}{2 h_{\theta}(\theta)} \operatorname{sign}\left(\frac{\partial h_{\theta}}{\partial \theta} \frac{\partial h_{\zeta}}{\partial \theta}-h_{\theta}(\theta) \frac{\partial^{2} h_{\zeta}}{\partial \theta^{2}}\right) \\
& \times\left\{\frac{1}{2} \ln \left[\left(\frac{h_{\theta}(\theta)}{8 h_{\zeta}(\theta)}\right) 2 \sin \left(\frac{\theta-\theta^{\prime}}{2}\right)\right]^{2}+1\right. \\
& \left.+\frac{h_{\zeta}(\theta) / h_{\theta}(\theta)}{h_{\theta}^{2}(\theta)-\left(\partial h_{\zeta} / \partial \theta\right)^{2}}\left(\frac{\partial h_{\theta}}{\partial \theta} \frac{\partial h_{\zeta}}{\partial \theta}-h_{\theta}(\theta) \frac{\partial^{2} h_{\zeta}}{\partial \theta^{2}}\right)\right\} .
\end{aligned}
$$

Therefore, the singularity of the normal component of the Green's function gradient can be handled introducing the dimensionless auxiliary function

$$
n\left(\theta ; \theta^{\prime}\right)=\widehat{n} \cdot \nabla G\left(\theta ; \theta^{\prime}\right)-\lim _{\theta^{\prime} \rightarrow \theta}\left[\widehat{n} \cdot \nabla G\left(\theta ; \theta^{\prime}\right)\right] \underset{\theta^{\prime} \rightarrow \theta}{\rightarrow} 0 .
$$

It follows that the coefficients $N_{m n}^{(s, s)}, N_{m n}^{(s, a)}, N_{m n}^{(a, s)}$ and $N_{m n}^{(a, a)}$ can be written with the singularities analytically removed, in a form suitable for numerical calculation:

$$
\begin{aligned}
& N_{m n}^{(s, s)}= \\
& \left\langle\left(\frac{h_{\zeta}(\theta)}{2 h_{\theta}(\theta)} \cos m \theta-\left\langle n\left(\theta ; \theta^{\prime}\right) \cos m \theta^{\prime}\right\rangle_{\theta^{\prime}}\right) \cos n \theta\right\rangle_{\theta} \\
& +\left\langle\frac{\sqrt{h_{\theta}^{2}(\theta)-\left(\partial h_{\zeta} / \partial \theta\right)^{2}}}{2 h_{\theta}(\theta)} \operatorname{sign}\left(\frac{\partial h_{\theta}}{\partial \theta} \frac{\partial h_{\zeta}}{\partial \theta}-h_{\theta}(\theta) \frac{\partial^{2} h_{\zeta}}{\partial \theta^{2}}\right)\right. \\
& \left.\times\left[\ln \left(\frac{8 h_{\zeta}(\theta)}{h_{\theta}(\theta)}\right)-1-\frac{h_{\zeta}(\theta) / h_{\theta}(\theta)}{h_{\theta}^{2}(\theta)-\left(\partial h_{\zeta} / \partial \theta\right)^{2}}\left(\frac{\partial h_{\theta}}{\partial \theta} \frac{\partial h_{\zeta}}{\partial \theta}-h_{\theta}(\theta) \frac{\partial^{2} h_{\zeta}}{\partial \theta^{2}}\right)\right] \cos n \theta\right\rangle_{\theta} \delta_{m, 0} \\
& +\left\langle\frac{\sqrt{h_{\theta}^{2}(\theta)-\left(\partial h_{\zeta} / \partial \theta\right)^{2}}}{2 h_{\theta}(\theta)} \operatorname{sign}\left(\frac{\partial h_{\theta}}{\partial \theta} \frac{\partial h_{\zeta}}{\partial \theta}-h_{\theta}(\theta) \frac{\partial^{2} h_{\zeta}}{\partial \theta^{2}}\right) \cos m \theta \cos n \theta\right\rangle_{\theta} \frac{1-\delta_{m, 0}}{2 m} \\
& N_{m n}^{(s, a)}= \\
& \left\langle\left(\frac{h_{\zeta}(\theta)}{2 h_{\theta}(\theta)} \sin m \theta-\left\langle n\left(\theta ; \theta^{\prime}\right) \sin m \theta^{\prime}\right\rangle_{\theta^{\prime}}\right) \cos n \theta\right\rangle_{\theta} \\
& +\left\langle\frac{\sqrt{h_{\theta}^{2}(\theta)-\left(\partial h_{\zeta} / \partial \theta\right)^{2}}}{2 h_{\theta}(\theta)} \operatorname{sign}\left(\frac{\partial h_{\theta}}{\partial \theta} \frac{\partial h_{\zeta}}{\partial \theta}-h_{\theta}(\theta) \frac{\partial^{2} h_{\zeta}}{\partial \theta^{2}}\right) \sin m \theta \cos n \theta\right\rangle_{\theta} \frac{1-\delta_{m, 0}}{2 m},
\end{aligned}
$$




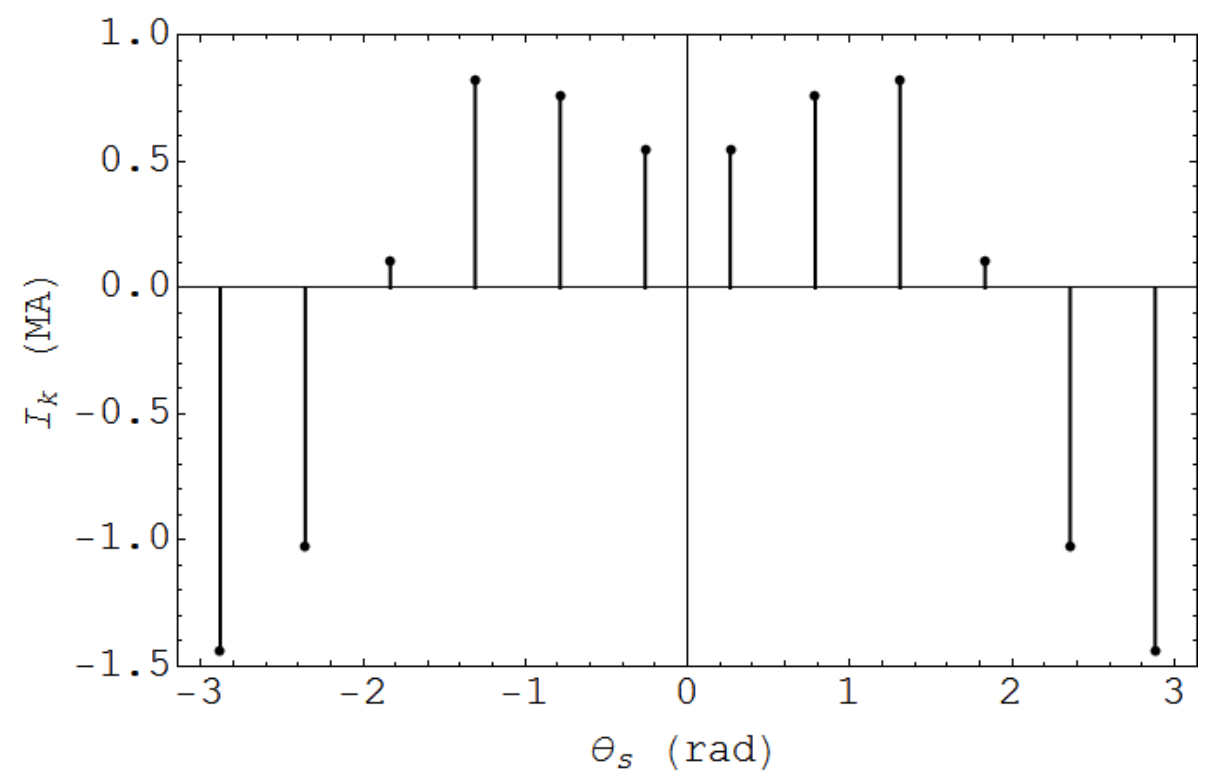

Figure 6: Distribution of currents in the set of external equilibrium coils shown in Fig. 1.

$$
\begin{aligned}
& N_{m n}^{(a, s)}= \\
& \left\langle\left(\frac{h_{\zeta}(\theta)}{2 h_{\theta}(\theta)} \cos m \theta-\left\langle n\left(\theta ; \theta^{\prime}\right) \cos m \theta^{\prime}\right\rangle_{\theta^{\prime}}\right) \sin n \theta\right\rangle_{\theta} \\
& +\left\langle\frac{\sqrt{h_{\theta}^{2}(\theta)-\left(\partial h_{\zeta} / \partial \theta\right)^{2}}}{2 h_{\theta}(\theta)} \operatorname{sign}\left(\frac{\partial h_{\theta}}{\partial \theta} \frac{\partial h_{\zeta}}{\partial \theta}-h_{\theta}(\theta) \frac{\partial^{2} h_{\zeta}}{\partial \theta^{2}}\right)\right. \\
& \left.\times\left[\ln \left(\frac{8 h_{\zeta}(\theta)}{h_{\theta}(\theta)}\right)-1-\frac{h_{\zeta}(\theta) / h_{\theta}(\theta)}{h_{\theta}^{2}(\theta)-\left(\partial h_{\zeta} / \partial \theta\right)^{2}}\left(\frac{\partial h_{\theta}}{\partial \theta} \frac{\partial h_{\zeta}}{\partial \theta}-h_{\theta}(\theta) \frac{\partial^{2} h_{\zeta}}{\partial \theta^{2}}\right)\right] \sin n \theta\right\rangle_{\theta} \delta_{m, 0} \\
& +\left\langle\frac{\sqrt{h_{\theta}^{2}(\theta)-\left(\partial h_{\zeta} / \partial \theta\right)^{2}}}{2 h_{\theta}(\theta)} \operatorname{sign}\left(\frac{\partial h_{\theta}}{\partial \theta} \frac{\partial h_{\zeta}}{\partial \theta}-h_{\theta}(\theta) \frac{\partial^{2} h_{\zeta}}{\partial \theta^{2}}\right) \cos m \theta \sin n \theta\right\rangle_{\theta} \frac{1-\delta_{m, 0}}{2 m} \\
& N_{m n}^{(a, a)}= \\
& \left\langle\left(\frac{h_{\zeta}(\theta)}{2 h_{\theta}(\theta)} \sin m \theta-\left\langle n\left(\theta ; \theta^{\prime}\right) \sin m \theta^{\prime}\right\rangle_{\theta^{\prime}}\right) \sin n \theta\right\rangle_{\theta} \\
& +\left\langle\frac{\sqrt{h_{\theta}^{2}(\theta)-\left(\partial h_{\zeta} / \partial \theta\right)^{2}}}{2 h_{\theta}(\theta)} \operatorname{sign}\left(\frac{\partial h_{\theta}}{\partial \theta} \frac{\partial h_{\zeta}}{\partial \theta}-h_{\theta}(\theta) \frac{\partial^{2} h_{\zeta}}{\partial \theta^{2}}\right) \sin m \theta \sin n \theta\right\rangle_{\theta} \frac{1-\delta_{m, 0}}{2 m} .
\end{aligned}
$$

Figure 6 shows the distribution of currents in the external coils necessary to establish the equilibrium shown in Fig. 1, also providing the flux to drive the toroidal current $I_{T, s}$ in the containing shell. This set of coils gives an excellent fit to the total poloidal flux distribution on the containing shell, but about twice the number of coils is needed if the same fit quality is required with respect to the maintaining magnetic field.

Collecting all previous results in a graphical form, Figs. 7, 8 and 9 show the total poloidal flux with respect to the symmetry axis in perspective view, in front view and in top orthographic projection, respectively, for the symmetric equilibrium shown in Fig. 1. The sharp change in the gradient of the poloidal flux corresponds to the containing shell contour. If the toroidal surface current density $K_{T, s}$ is distributed over an annulus repre- 


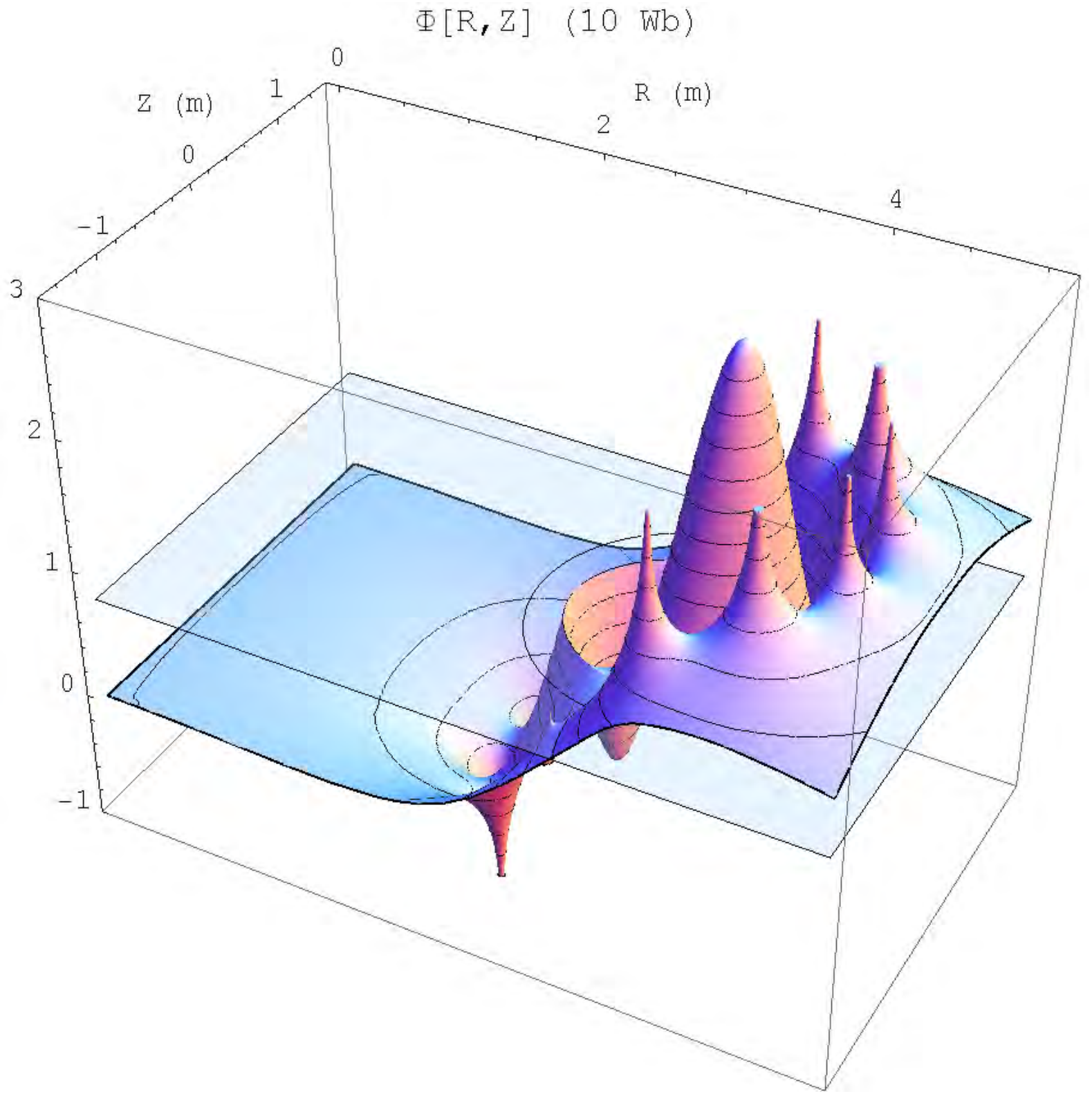

Figure 7: Perspective view of the total poloidal flux with respect to the symmetry axis for the equilibrium shown in Fig. 1. 


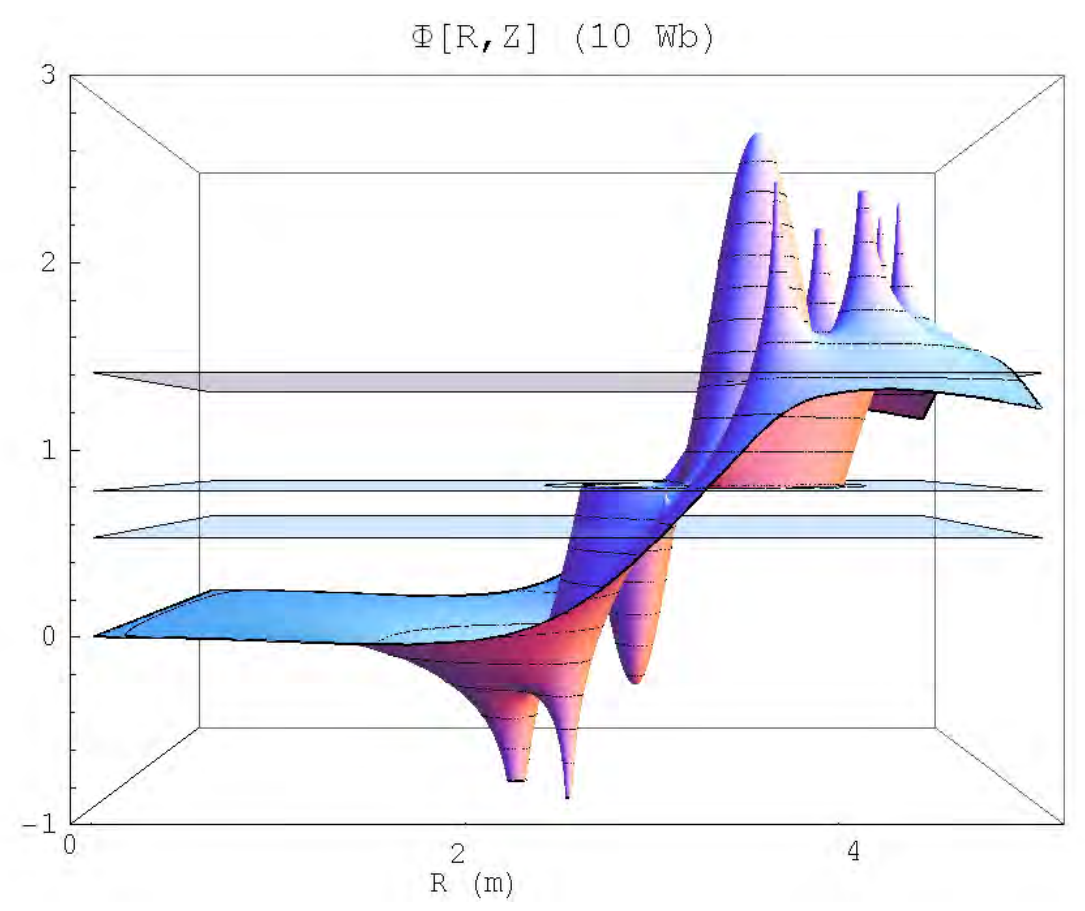

Figure 8: Front view of the total poloidal flux for the equilibrium shown in Figs. 1 and 7 . The horizontal planes correspond, from top to bottom, to the constant flux values $\Phi_{j}(0)=14.180 \mathrm{~Wb}, \Phi_{s}(0)=7.830 \mathrm{~Wb}$ and $\Phi_{i}(0)=5.330 \mathrm{~Wb}$.

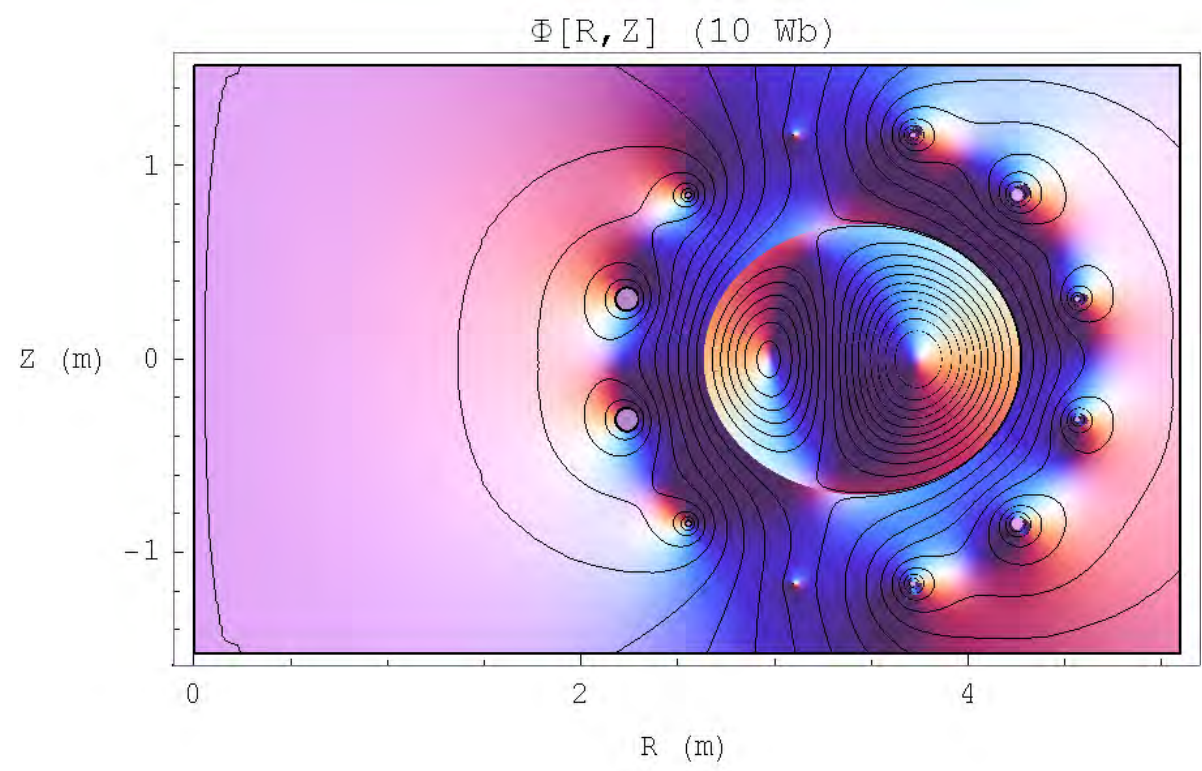

Figure 9: Top orthographic projection of the total poloidal flux for the equilibrium shown in Figs. 1, 7 and 8. 
senting the outer plasma region this change becomes gradual. Of course, the containing contour can be considered as a virtual shell for the maintaining field, instead of a true current carrying shell, but only if the total toroidal shell current $I_{T, s}$ vanishes.

\section{Magnetohydrodynamic stability analysis}

The role of reconnection in the formation of current holes has been studied before by means of resistive magnetohydrodynamic (MHD) simulations in toroidal geometry [21]. It was argued that reconnection events redistribute the current in the plasma core holding the current density near zero, preventing strong reversal and islands formation in the center of the discharge. However, no indication of the required MHD instabilities have been observed in the experiments [8]. Furthermore, as briefly discussed in the Introduction, the AC experiments clearly show that current reversal is possible so that the current reversal issue as a whole remains unresolved. In this section it is assumed that the strong current reversal equilibrium with two islands studied in the previous sections as been established. Then, a preliminary MHD stability analysis of the reversed current configuration is conducted by means of the energy principle.

The energy stored in the islands equilibrium is formed by the poloidal and toroidal magnetic field energies in the shell and islands circuits, plus the energy due to quasistatic work of the islands. The poloidal magnetic field energy has contributions from the selfenergies, $W_{P, i i}, W_{P, j j}, W_{P, s s}$, and the mutual energies, $W_{P, i j}, W_{P, i s}, W_{P, j s}$ of the islands and shell circuits. The toroidal magnetic field energy includes the energies stored in the islands, $W_{T, i}+W_{T, 0, i}$ and $W_{T, j}+W_{T, 0, j}$, due to both the intrinsic and external toroidal magnetic fields. Finally, the energies due to quasistatic work done by the islands is denoted by $W_{Q S, i}$ and $W_{Q S, j}$. All these contributions are evaluated in the following.

The poloidal self magnetic field energy of the island $i$ is

$$
W_{P, i i}\left(a_{i}\right)=\frac{1}{2}\left(L_{i, \mathrm{ext}}\left(a_{i}\right)+L_{i, \mathrm{int}}\left(a_{i}\right)\right) I_{T, i}^{2}\left(a_{i}\right),
$$

where

$$
\begin{aligned}
L_{i, \mathrm{ext}}\left(a_{i}\right) & =L_{i i, 0}^{(s)}=\frac{1}{K_{i}\left(a_{i}\right)}\left\langle\left\langle\left(\frac{h_{\theta}^{2}\left(a_{i}, \theta_{i}^{\prime}\right)}{\sqrt{g}\left(a_{i}, \theta_{i}^{\prime}\right)}\right) G\left(\theta_{i} ; \theta_{i}^{\prime}\right)\right\rangle_{\theta_{i}^{\prime}}\right\rangle_{\theta_{i}}, \\
L_{i, \text { int }}\left(a_{i}\right) & =\frac{1}{I_{T, i}^{2}\left(a_{i}\right)} \int_{0}^{a_{i}} \frac{I_{T, i}^{2}(\rho)}{K_{i}(\rho)} d \rho,
\end{aligned}
$$

denote the external and internal inductances of the island.

The poloidal self magnetic field energy of the shell $s$ is

$$
\begin{aligned}
W_{P, s s}= & \frac{\mu_{0}}{2} \oint_{\ell_{s}} \oint_{\ell_{s}^{\prime}} K_{T, s}\left(\theta_{s}\right) K_{T, s}\left(\theta_{s}^{\prime}\right) G\left(\theta_{s} ; \theta_{s}^{\prime}\right) d \ell\left(\theta_{s}^{\prime}\right) d \ell\left(\theta_{s}\right) \\
= & \frac{1}{2} L_{s s, 00}^{(s)} I_{T, s}^{2}+I_{T, s} \sum_{m=1}^{n_{s}} L_{s s, m 0}^{(s)} I_{m}^{(s)} \\
& +\frac{1}{2} \sum_{m=1}^{n_{s}} L_{s s, m m}^{(s)}\left(I_{m}^{(s)}\right)^{2}+2 \sum_{m=2}^{n_{s}} \sum_{n=1}^{m-1} L_{s s, m n}^{(s)} I_{m}^{(s)} I_{n}^{(s)} \\
& +\frac{1}{2} \sum_{m=1}^{n_{s}} L_{s s, m m}^{(a)}\left(I_{m}^{(a)}\right)^{2}+2 \sum_{m=2}^{n_{s}} \sum_{n=1}^{m-1} L_{s s, m n}^{(a)} I_{m}^{(a)} I_{n}^{(a)}
\end{aligned}
$$


The poloidal mutual magnetic field energy between islands $i$ and $j$ is

$$
\begin{aligned}
W_{P, i j}\left(a_{i}, a_{j}\right) & =\mu_{0} \oint_{\ell_{j}} \oint_{\ell_{i}} K_{T, j}\left(a_{j}, \theta_{j}\right) K_{T, i}\left(a_{i}, \theta_{i}\right) G\left(\theta_{j} ; \theta_{i}\right) d \ell\left(\theta_{i}\right) d \ell\left(\theta_{j}\right) \\
& =M_{i j} I_{T, i}\left(a_{i}\right) I_{T, j}\left(a_{j}\right)=M_{j i} I_{T, i}\left(a_{i}\right) I_{T, j}\left(a_{j}\right) .
\end{aligned}
$$

The poloidal mutual magnetic field energy between the island $i$ and the shell $s$ is

$$
\begin{aligned}
W_{P, i s}\left(a_{i}\right) & =\mu_{0} \oint_{\ell_{s}} \oint_{\ell_{i}} K_{T, s}\left(\theta_{s}\right) K_{T, i}\left(a_{i}, \theta_{i}\right) G\left(\theta_{s} ; \theta_{i}\right) d \ell\left(\theta_{i}\right) d \ell\left(\theta_{s}\right) \\
& =\left(M_{s i, 0}^{(s)} I_{T, s}+\sum_{m=1}^{n_{s}}\left(M_{s i, m}^{(s)} I_{m}^{(s)}+M_{s i, m}^{(a)} I_{m}^{(a)}\right)\right) I_{T, i}\left(a_{i}\right) \\
& =\left(M_{i s, 0}^{(s)} I_{T, s}+\sum_{m=1}^{n_{s}}\left(M_{i s, m}^{(s)} I_{m}^{(s)}+M_{i s, m}^{(a)} I_{m}^{(a)}\right)\right) I_{T, i}\left(a_{i}\right) \\
& =M_{s i} I_{T, s} I_{T, i}\left(a_{i}\right)=M_{i s} I_{T, s} I_{T, i}\left(a_{i}\right) .
\end{aligned}
$$

The total toroidal magnetic field energy stored in the island $i$ is

$$
W_{T, i}\left(a_{i}\right)+W_{T, 0, i}\left(a_{i}\right)=\int_{0}^{a_{i}} \frac{L_{i}(\rho)}{d L_{i} / d \rho}\left(\frac{I_{T, i}(\rho)}{K_{i}(\rho)} \frac{d I_{T, i}}{d \rho}+\frac{d V_{i}}{d \rho} \frac{d p_{i}}{d \rho}\right) d \rho+W_{T, 0, i}\left(a_{i}\right),
$$

where the second term refers to the magnetic energy due to the external toroidal magnetic field inside the empty island cavity, which does not vary in a fixed boundary situation

$$
W_{T, 0, i}\left(a_{i}\right)=\frac{1}{2} L_{i}\left(a_{i}\right) I_{i}^{2}\left(a_{i}\right)=\frac{1}{2} L_{i}\left(a_{i}\right)\left(\frac{2 \pi R_{0} B_{0}}{\mu_{0}}\right)^{2} .
$$

In the same way, the toroidal magnetic field energy due to $B_{0}$ and stored in the empty containing shell is given by (note that there is no poloidal current flowing in the shell)

$$
W_{T, 0, s}=\frac{1}{2} L_{s}\left(a_{s}\right) I_{s}^{2}\left(a_{s}\right)=\frac{1}{2} L_{s}\left(a_{s}\right)\left(\frac{2 \pi R_{0} B_{0}}{\mu_{0}}\right)^{2}
$$

where

$$
L_{s}\left(a_{s}\right)=-\frac{\mu_{0}}{2 \pi} \int_{0}^{2 \pi}\left(\frac{Z_{s}(\theta)}{R_{s}(\theta)} \frac{\partial R_{s}}{\partial \theta}\right) d \theta .
$$

Finally, the stored energy due to the quasistatic (reversible) mechanical work done by expansion of the island $i$ is

$$
W_{Q S, i}\left(a_{i}\right)=\int_{0}^{a_{i}} p_{i}(\rho) \frac{d V_{i}}{d \rho} d \rho
$$

The total energy stored in the islands-shell system, excluding the interaction of the shell with the external coils and the toroidal magnetic field energy stored in the empty containing shell, is given by the sum

$$
W_{\text {total }}=W_{P, i i}+W_{P, j j}+W_{P, s s}+W_{P, i j}+W_{P, i s}+W_{P, j s}+W_{T, i}+W_{T, j}+W_{Q S, i}+W_{Q S, j} .
$$


Now, the equilibrium is perturbed by a small rotation $\omega$ around the shell center, such that the major vertical positions of the islands become

$$
\begin{aligned}
& Z_{0, i}\left(a_{i}\right)=\left(R_{0, i}\left(a_{i}\right)-R_{0, s}\right) \tan \omega \\
& Z_{0, j}\left(a_{i}\right)=\left(R_{0, j}\left(a_{j}\right)-R_{0, s}\right) \tan \omega .
\end{aligned}
$$

During this tilting motion the total toroidal currents $I_{T, i}(0)=-3.92 \mathrm{MA}, I_{T, j}(0)=$ 4.52 MA flowing in the islands, as well as the total poloidal fluxes $\Phi_{P, i}(0)=-10.010 \mathrm{~Wb}$, $\Phi_{P, j}(0)=15.750 \mathrm{~Wb}$ contained by the islands, are assumed constant. It is also assumed that the thermodynamic pressure in the islands varies according to the quasistatic adiabatic equation of state:

$$
p(0) V(a)^{\gamma}=\text { constant } \quad \gamma=5 / 3 \text {. }
$$

The remaining equilibrium parameters are calculated by the method of matching moments described in Section 4. The results of these calculations are listed in Tables 2 and 3 for island $i$, Tables 4 and 5 for island $j$, and Tables 6 and 7 for the containing shell $s$. The symmetric equilibrium values which correspond to $\omega=0^{\circ}$ are listed in Table 1 . These results show the occurrence of a critical point at a maximum rotation angle $\omega \cong 3.9^{\circ}$, resulting in a bifurcation of the equilibrium for $0 \leqslant \omega \lesssim 3.9^{\circ}$. The low-energy equilibrium branch corresponds to the direct tilt (forward motion) extending from the symmetric equilibrium solution $(\omega=0)$ up to the critical point. Tables 2 and 4 indicate that both islands are compressed during the direct tilt. Actually, the inner island suffers a mild compression with a maximum pressure $p_{0, i}=13.97 \mathrm{~Pa}$ occurring near the critical point (more precisely for $\omega \cong 3.8^{\circ}$ ) and a slow expansion afterwards. The high-energy equilibrium branch corresponding to the reverse tilt (backward motion) is characterized by an expansion of the inner island and a strong compression of the outer one, which collapses into a nonsymmetric filament with relatively high plasma pressure. Figures 10 and 11 illustrate the change of the islands shape during the direct and reverse tilting displacements, respectively. Of course, a specular set of equilibria with respect to the equatorial plane $Z=0$ is obtained changing the sign of both $\omega$ and the antisymmetric coefficients in the spectral representation of the islands.

Figures 12 to 18 show the changes in the energy terms of the islands-shell system during the direct and reverse tilting displacements, along both equilibrium branches. Initially, Fig. 12 shows the poloidal self magnetic field energies $W_{P, i i}$ and $W_{P, j j}$ stored in the islands: $W_{P, i i}$ varies to a small extent around $75 \mathrm{MJ}$, with constant total toroidal current $I_{T, i}$ and small changes in the geometry; in the same way the energy $W_{P, j j}$ presents a relatively small variation in the direct tilt branch but varies considerably in the reverse tilt, due to the large increase in the inductance of the outer island during the compression process, although keeping constant total toroidal current $I_{T, j}$. The dashed lines in Fig. 12 correspond to the large-aspect-ratio approximation

$$
W_{P, \text { self }}(a) \cong \frac{\mu_{0} R_{m}}{2}\left[\ln \left(\frac{8 R_{m}}{a}\right)-2+\frac{\ell_{i}}{2}\right] I_{T}^{2}(a) .
$$

Here $\ell_{i}$ is the internal inductance defined by

$$
\ell_{i}=\frac{2}{\mu_{0} R_{m}} \frac{1}{I_{T}^{2}(a)} \int_{0}^{a} \frac{I_{T}^{2}(\rho)}{K(\rho)} d \rho \cong \frac{2 \kappa_{m}}{\kappa_{m}^{2}+1} \ln \left[\exp \left(\frac{1}{2}\right)+\frac{\exp \left(\gamma_{E}\right)}{2} \alpha_{j}\right]
$$




\begin{tabular}{|l||l|l|l|l|l|l|}
\hline Island $i$ & $\omega=1^{\circ}$ & $\omega=2^{\circ}$ & $\omega=3^{\circ}$ & $\omega=3.3^{\circ}$ & $\omega=3.6^{\circ}$ & $\omega=3.9^{\circ}$ \\
\hline \hline$R_{0, i}(\mathrm{~m})$ & 2.954 & 2.954 & 2.955 & 2.956 & 2.957 & 2.958 \\
\hline$Z_{0, i}(\mathrm{~mm})$ & -8.67 & -17.3 & -25.9 & -28.5 & -31.0 & -33.5 \\
\hline$a_{i}(\mathrm{~m})$ & 0.263 & 0.263 & 0.262 & 0.262 & 0.261 & 0.261 \\
\hline$e_{i}$ & 1.693 & 1.693 & 1.692 & 1.691 & 1.690 & 1.689 \\
\hline$S_{2, i}$ & -0.0715 & -0.0717 & -0.0718 & -0.0717 & -0.0719 & -0.0731 \\
\hline$A_{2, i}$ & -0.0223 & -0.0445 & -0.0664 & -0.0728 & -0.0791 & -0.0846 \\
\hline$S_{3, i}$ & -0.0135 & -0.0137 & -0.0141 & -0.0143 & -0.0146 & -0.0155 \\
\hline$A_{3, i}$ & -0.000668 & -0.00140 & -0.00226 & -0.00257 & -0.00293 & -0.00354 \\
\hline$R_{m, i}(\mathrm{~m})$ & 2.966 & 2.967 & 2.968 & 2.969 & 2.970 & 2.971 \\
\hline$Z_{m, i}(\mathrm{~mm})$ & -6.69 & -13.2 & -19.2 & -20.9 & -22.6 & -24.3 \\
\hline$\kappa_{m, i}$ & 1.554 & 1.543 & 1.526 & 1.519 & 1.513 & 1.507 \\
\hline$p_{0, i}(\mathrm{kPa})$ & 13.38 & 13.48 & 13.72 & 13.82 & 13.93 & 13.92 \\
\hline$\Phi_{i}(\mathrm{~Wb})$ & 5.322 & 5.292 & 5.223 & 5.191 & 5.152 & 5.111 \\
\hline
\end{tabular}

Table 2: Parameters of the inner island $i$ for a small direct tilt around the containing shell center (low-energy equilibrium branch).

\begin{tabular}{|l||l|l|l|l|l|l|}
\hline Island $i$ & $\omega=3.9^{\circ}$ & $\omega=3.6^{\circ}$ & $\omega=3.3^{\circ}$ & $\omega=3^{\circ}$ & $\omega=2^{\circ}$ & $\omega=1^{\circ}$ \\
\hline \hline$R_{0, i}(\mathrm{~m})$ & 2.960 & 2.962 & 2.963 & 2.965 & 2.970 & 2.980 \\
\hline$Z_{0, i}(\mathrm{~mm})$ & -33.4 & -30.7 & -28.1 & -25.4 & -16.8 & -8.21 \\
\hline$a_{i}(\mathrm{~m})$ & 0.262 & 0.265 & 0.267 & 0.269 & 0.276 & 0.286 \\
\hline$e_{i}$ & 1.691 & 1.696 & 1.699 & 1.701 & 1.706 & 1.710 \\
\hline$S_{2, i}$ & -0.0754 & -0.0803 & -0.0836 & -0.0865 & -0.0958 & -0.110 \\
\hline$A_{2, i}$ & -0.0833 & -0.0749 & -0.0674 & -0.0603 & -0.0382 & -0.0178 \\
\hline$S_{3, i}$ & -0.0167 & -0.0189 & -0.0203 & -0.0214 & -0.0251 & -0.0305 \\
\hline$A_{3, i}$ & -0.00393 & -0.00423 & -0.00421 & -0.00409 & -0.00325 & -0.00203 \\
\hline$R_{m, i}(\mathrm{~m})$ & 2.973 & 2.976 & 2.978 & 2.980 & 2.987 & 2.999 \\
\hline$Z_{m, i}(\mathrm{~mm})$ & -24.5 & -23.0 & -21.3 & -19.6 & -13.3 & -6.61 \\
\hline$\kappa_{m, i}$ & 1.511 & 1.523 & 1.532 & 1.540 & 1.558 & 1.566 \\
\hline$p_{0, i}(\mathrm{kPa})$ & 13.67 & 13.03 & 12.57 & 12.17 & 10.95 & 9.441 \\
\hline$\Phi_{i}(\mathrm{~Wb})$ & 5.121 & 5.183 & 5.232 & 5.275 & 5.403 & 5.571 \\
\hline
\end{tabular}

Table 3: Parameters of the inner island $i$ for a small reverse tilt around the containing shell center (high-energy equilibrium branch). 


\begin{tabular}{|l||l|l|l|l|l|l|}
\hline Island $j$ & $\omega=1^{\circ}$ & $\omega=2^{\circ}$ & $\omega=3^{\circ}$ & $\omega=3.3^{\circ}$ & $\omega=3.6^{\circ}$ & $\omega=3.9^{\circ}$ \\
\hline \hline$R_{0, j}(\mathrm{~m})$ & 3.749 & 3.749 & 3.749 & 3.749 & 3.750 & 3.751 \\
\hline$Z_{0, j}(\mathrm{~mm})$ & 5.21 & 10.4 & 15.7 & 17.3 & 18.8 & 20.5 \\
\hline$a_{j}(\mathrm{~m})$ & 0.379 & 0.373 & 0.360 & 0.353 & 0.343 & 0.320 \\
\hline$e_{j}$ & 1.252 & 1.245 & 1.230 & 1.222 & 1.210 & 1.184 \\
\hline$S_{2, j}$ & 0.0570 & 0.0531 & 0.0457 & 0.0423 & 0.0377 & 0.0288 \\
\hline$A_{2, j}$ & 0.0127 & 0.0258 & 0.0402 & 0.0452 & 0.0511 & 0.0602 \\
\hline$S_{3, j}$ & -0.0161 & -0.0145 & -0.0116 & -0.0103 & -0.00872 & -0.00591 \\
\hline$A_{3, j}$ & -0.00519 & -0.0100 & -0.0142 & -0.0152 & -0.0161 & -0.0167 \\
\hline$R_{m, j}(\mathrm{~m})$ & 3.738 & 3.739 & 3.741 & 3.742 & 3.744 & 3.747 \\
\hline$Z_{m, j}(\mathrm{~mm})$ & 1.22 & 2.37 & 3.32 & 3.51 & 3.60 & 3.30 \\
\hline$\kappa_{m, j}$ & 1.212 & 1.205 & 1.190 & 1.183 & 1.172 & 1.149 \\
\hline$p_{0, j}(\mathrm{kPa})$ & 13.63 & 14.62 & 17.02 & 18.42 & 20.72 & 27.22 \\
\hline$\Phi_{j}(\mathrm{~Wb})$ & 14.300 & 14.690 & 15.560 & 16.010 & 16.700 & 18.320 \\
\hline
\end{tabular}

Table 4: Parameters of the outer island $j$ for a small direct tilt around the containing shell center (low-energy equilibrium branch).

\begin{tabular}{|l||l|l|l|l|l|l|}
\hline Island $j$ & $\omega=3.9^{\circ}$ & $\omega=3.6^{\circ}$ & $\omega=3.3^{\circ}$ & $\omega=3^{\circ}$ & $\omega=2^{\circ}$ & $\omega=1^{\circ}$ \\
\hline \hline$R_{0, j}(\mathrm{~m})$ & 3.752 & 3.756 & 3.759 & 3.762 & 3.772 & 3.788 \\
\hline$Z_{0, j}(\mathrm{~mm})$ & 20.61 & 19.25 & 17.81 & 16.34 & 11.25 & 5.90 \\
\hline$a_{j}(\mathrm{~m})$ & 0.299 & 0.264 & 0.241 & 0.221 & 0.155 & 0.0826 \\
\hline$e_{j}$ & 1.160 & 1.124 & 1.103 & 1.085 & 1.041 & 1.012 \\
\hline$S_{2, j}$ & 0.0221 & 0.0140 & 0.0101 & 0.00745 & 0.00231 & 0.000338 \\
\hline$A_{2, j}$ & 0.0657 & 0.0713 & 0.0736 & 0.0751 & 0.0782 & 0.0833 \\
\hline$S_{3, j}$ & -0.00403 & -0.00206 & -0.00127 & -0.000792 & -0.000101 & 0.0000154 \\
\hline$A_{3, j}$ & -0.0165 & -0.0158 & -0.0153 & -0.0149 & -0.0142 & -0.0118 \\
\hline$R_{m, j}(\mathrm{~m})$ & 3.750 & 3.755 & 3.759 & 3.762 & 3.773 & 3.788 \\
\hline$Z_{m, j}(\mathrm{~mm})$ & 2.72 & 1.62 & 0.96 & 0.44 & -0.62 & -0.94 \\
\hline$\kappa_{m, j}$ & 1.129 & 1.100 & 1.083 & 1.069 & 1.033 & 1.010 \\
\hline$p_{0, j}(\mathrm{kPa})$ & 35.62 & 56.87 & 79.45 & 109.7 & 385.0 & $3.256 \times 10^{3}$ \\
\hline$\Phi_{j}(\mathrm{~Wb})$ & 19.940 & 22.800 & 24.860 & 26.870 & 34.750 & 48.300 \\
\hline
\end{tabular}

Table 5: Parameters of the outer island $j$ for a small reverse tilt around the containing shell center (high-energy equilibrium branch). 


\begin{tabular}{|l||l|l|l|l|l|l|}
\hline Shell $s$ & $\omega=1^{\circ}$ & $\omega=2^{\circ}$ & $\omega=3^{\circ}$ & $\omega=3.3^{\circ}$ & $\omega=3.6^{\circ}$ & $\omega=3.9^{\circ}$ \\
\hline \hline$I_{1}^{(s)}(\mathrm{MA})$ & -8.203 & -8.217 & -8.249 & -8.264 & -8.287 & -8.331 \\
\hline$I_{2}^{(s)}(\mathrm{MA})$ & 2.533 & 2.483 & 2.389 & 2.347 & 2.287 & 2.161 \\
\hline$I_{3}^{(s)}(\mathrm{kA})$ & 315.7 & 321.5 & 321.0 & 316.9 & 308.9 & 290.8 \\
\hline$I_{4}^{(s)}(\mathrm{kA})$ & -166.5 & -157.4 & -139.4 & -131.8 & -123.0 & -113.5 \\
\hline$I_{1}^{(a)}(\mathrm{kA})$ & 39.44 & 75.16 & 102.0 & 106.9 & 109.0 & 103.2 \\
\hline$I_{2}^{(a)}(\mathrm{kA})$ & -9.440 & -18.77 & -26.62 & -28.07 & -28.47 & -24.94 \\
\hline$I_{3}^{(a)}(\mathrm{kA})$ & -40.18 & -76.59 & -105.0 & -110.9 & -114.7 & -113.7 \\
\hline$I_{4}^{(a)}(\mathrm{kA})$ & 12.12 & 26.39 & 45.54 & 53.41 & 63.61 & 82.69 \\
\hline
\end{tabular}

Table 6: Fourier components of the surface current density in the containing shell $s$ for a small direct tilt of the islands around the shell center (low-energy equilibrium branch). The total poloidal flux on the shell remains constant at a value $\Phi_{s}=7.830 \mathrm{~Wb}$ defined by the fixed shape and constant total current $I_{T, s}=600 \mathrm{kA}$.

\begin{tabular}{|l||l|l|l|l|l|l|}
\hline Shell $s$ & $\omega=3.9^{\circ}$ & $\omega=3.6^{\circ}$ & $\omega=3.3^{\circ}$ & $\omega=3^{\circ}$ & $\omega=2^{\circ}$ & $\omega=1^{\circ}$ \\
\hline \hline$I_{1}^{(s)}(\mathrm{MA})$ & -8.367 & -8.415 & -8.443 & -8.466 & -8.528 & -8.575 \\
\hline$I_{2}^{(s)}(\mathrm{MA})$ & 2.047 & 1.873 & 1.766 & 1.674 & 1.385 & 0.9593 \\
\hline$I_{3}^{(s)}(\mathrm{kA})$ & 278.8 & 270.1 & 270.2 & 273.8 & 307.4 & 392.0 \\
\hline$I_{4}^{(s)}(\mathrm{kA})$ & -114.7 & -127.3 & -138.1 & -148.1 & -176.9 & -201.1 \\
\hline$I_{1}^{(a)}(\mathrm{kA})$ & 92.66 & 74.09 & 62.99 & 54.21 & 33.29 & 19.80 \\
\hline$I_{2}^{(a)}(\mathrm{kA})$ & -19.07 & -8.760 & -2.786 & 1.664 & 10.01 & 11.93 \\
\hline$I_{3}^{(a)}(\mathrm{kA})$ & -107.8 & -95.17 & -86.10 & -77.82 & -51.89 & -26.52 \\
\hline$I_{4}^{(a)}(\mathrm{kA})$ & 96.26 & 108.7 & 110.7 & 108.9 & 87.94 & 51.19 \\
\hline
\end{tabular}

Table 7: Fourier components of the surface current density in the containing shell $s$ for a small reverse tilt of the islands around the shell center (high-energy equilibrium branch). The total poloidal flux on the shell remains constant at a value $\Phi_{s}=7.830 \mathrm{~Wb}$ defined by the fixed shape and constant total current $I_{T, s}=600 \mathrm{kA}$. 

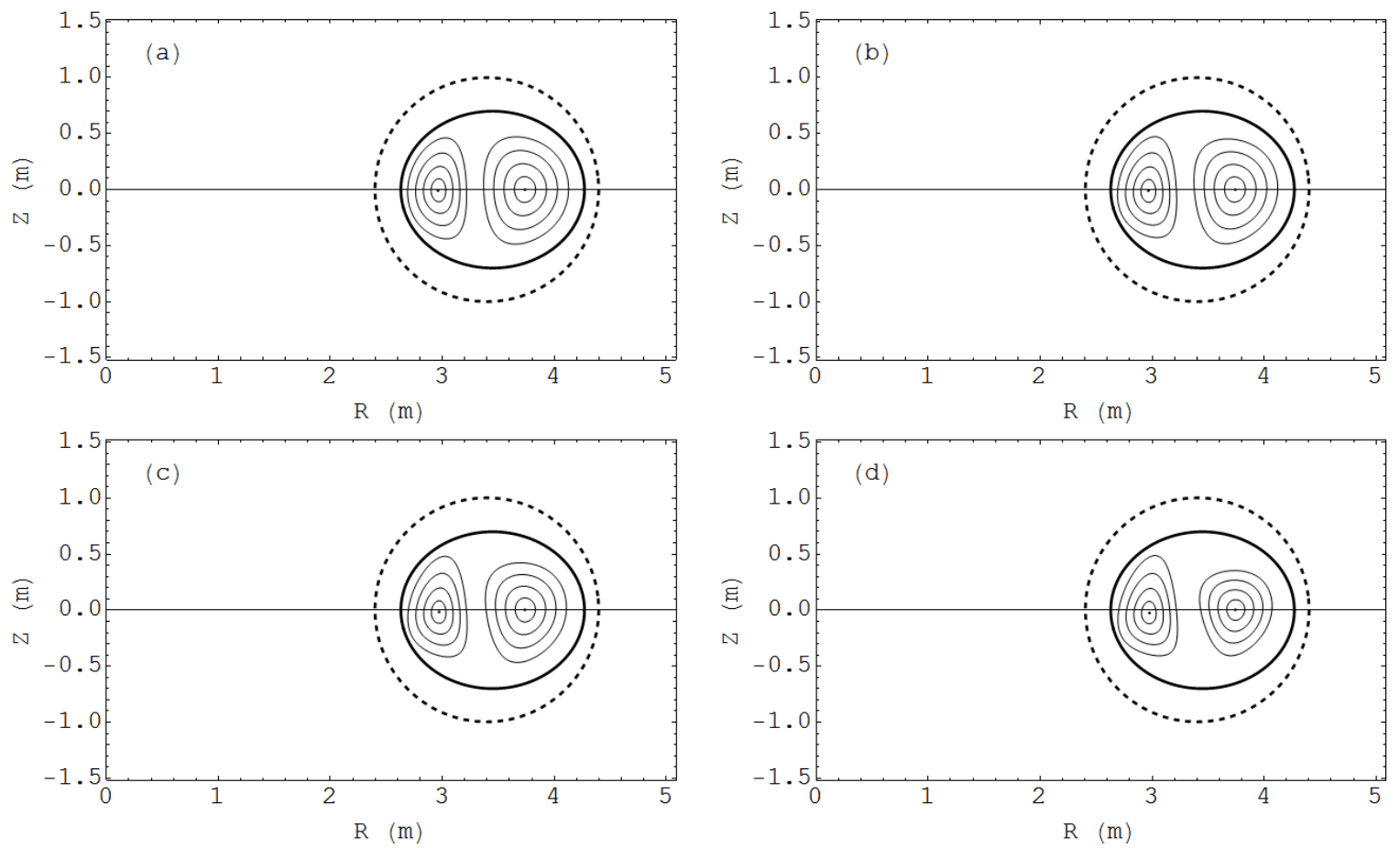

Figure 10: Sequence of islands equilibria for a direct tilt: (a) $\omega=1^{\circ}$; (b) $\omega=2^{\circ}$; (c) $\omega=3^{\circ}$; (d) $\omega=3.9^{\circ}$. This sequence corresponds to the low-energy equilibrium branch shown in Fig. 18.
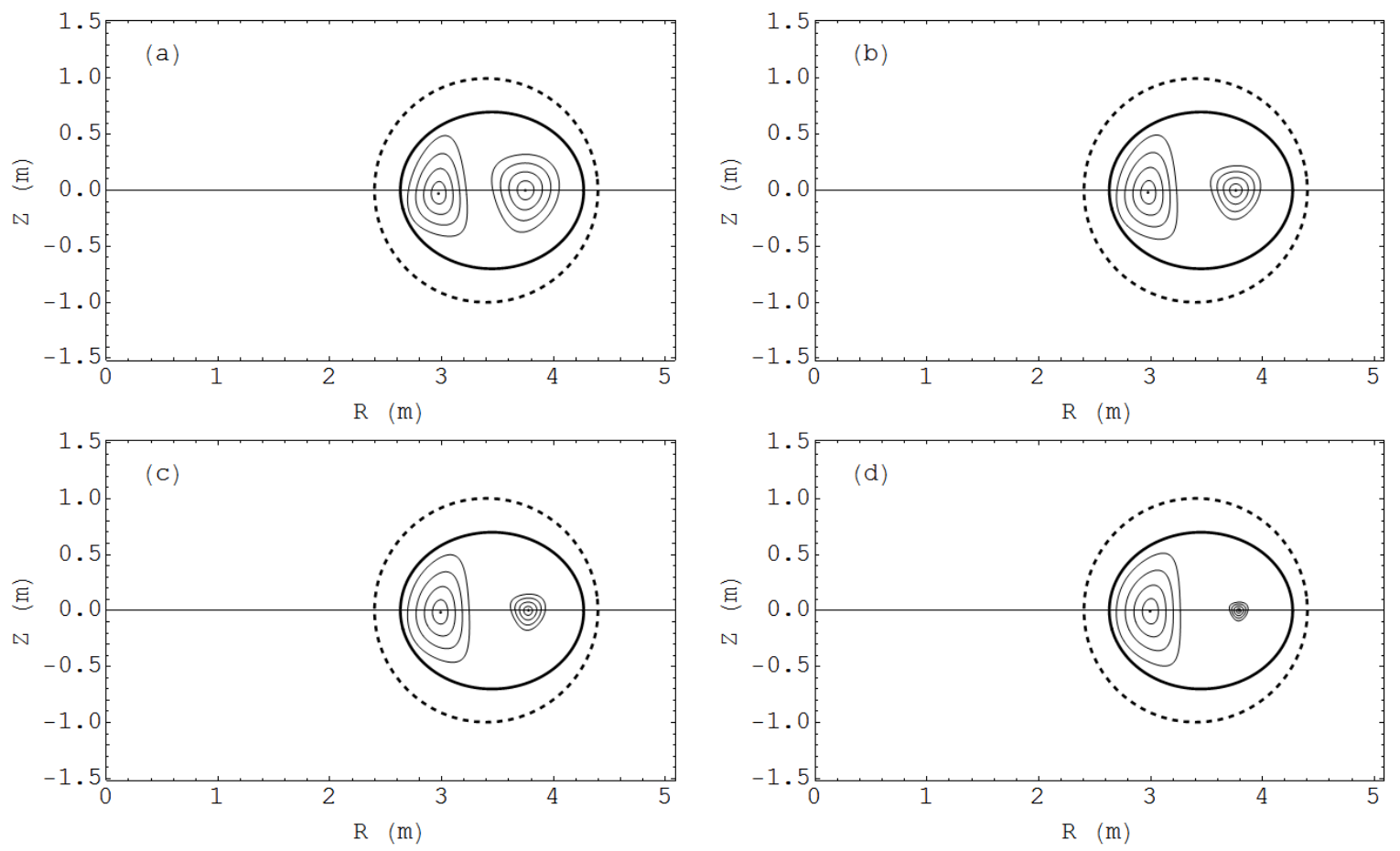

Figure 11: Sequence of islands equilibria for a reverse tilt: (a) $\omega=3.9^{\circ}$; (b) $\omega=3^{\circ}$; (c) $\omega=2^{\circ}$; (d) $\omega=1^{\circ}$. This sequence corresponds to the high-energy equilibrium branch shown in Fig. 18. 
where $\gamma_{E} \cong 0.577$ is the Euler's constant and $\alpha_{j}$ is the exponent in the binomial approximation of the flux-surface averaged toroidal current density

$$
j_{T}(\rho) \cong \frac{1+\alpha_{j}}{\pi a^{2} \kappa_{m}}\left(1-\frac{\rho^{2}}{a^{2}}\right)^{\alpha_{j}} I_{T}(a) \text { with } I_{T}(\rho) \cong\left[1-\left(1-\frac{\rho^{2}}{a^{2}}\right)^{1+\alpha_{j}}\right] I_{T}(a) .
$$

The total toroidal current profile used in this work can be fitted with $\alpha_{j} \cong 0.957$. Of course, the large-aspect-ratio approximation becomes very good in the final stages of compression of the outer island. Now, the poloidal self magnetic field energy $W_{P, s s}$ changes as shown in Fig. 13, due to a redistribution of the surface current density induced mostly by the collapsing external island. Figure 14 shows the change in the poloidal magnetic field energy $W_{P, i j}$ associated with the interaction between islands; the increasing negative magnetic energy corresponds to the repulsive force between the islands that are contained by the surface current induced in the shell. The dashed line corresponds to the interaction energy between two filaments

$$
W_{P, i j} \cong \mu_{0} \sqrt{R_{m, i} R_{m, j}}\left(\frac{\left(2-m_{i j}\right) K\left(m_{i j}\right)-2 E\left(m_{i j}\right)}{\sqrt{m_{i j}}}\right) I_{T, i} I_{T, j}
$$

where $K\left(m_{i j}\right)$ and $E\left(m_{i j}\right)$ are the complete elliptic integrals and

$$
m_{i j}=\frac{4 R_{m, i} R_{m, j}}{\left(R_{m, i}+R_{m, j}\right)^{2}+\left(Z_{m, i}-Z_{m, j}\right)^{2}}
$$

This formula gives a reasonable approximation of the mutual inductance effects. The changes in the poloidal mutual magnetic field energy terms $W_{P, i s}$ and $W_{P, j s}$, due to the repulsive interaction between the islands and the shell, are shown in Fig. 15. Next, as shown in Fig. 16, the intrinsic toroidal magnetic field energies $W_{T, i}$ and $W_{T, j}$ stored in the islands do not change significantly; in the final stages of the strong compression phase $W_{T, j}$ presents a small reduction due to the diamagnetic effect. Finally, Fig. 17 shows the changes in the quasistatic energy terms $W_{Q S, i}$ and $W_{Q S, j}$ : $W_{Q S, i}$ changes slightly around $21 \mathrm{~kJ} ; W_{Q S, j}$ increases significantly to the $1 \mathrm{MJ}$ level during the compression process though contributing very little to the total stored energy.

Figure 18 shows the total energy $W_{\text {total }}$ given by the sum of the energy contributions displayed in the previous figures. The quasistatic energy terms $W_{Q S, i}$ and $W_{Q S, j}$ have a negligible contribution. It turns out that the energy terms $W_{P, s s}, W_{P, i s}, W_{P, j s}, W_{T, i}$ and $W_{T, j}$ are not negligible, but in large part cancel out. Hence, Fig. 18 compares the total energy with the sum of the remaining contributions $W_{P, i i}, W_{P, j j}$ and $W_{P, i j}$. Also, the dashed line in Fig. 18 gives the combined sum of the large-aspect-ratio and filaments approximations given above. The coupling coefficient between the two islands varies from $0.59\left(\omega=0^{\circ}\right)$ to $0.56\left(\omega=3.9^{\circ}\right)$ along the low-energy branch, and down to $0.44\left(\omega=0.4^{\circ}\right)$ and lower values along the high-energy branch. Since $W_{P, i i}$ is practically constant, the changes in the total stored energy are dominated by the interplay between $W_{P, i j}$ and $W_{P, j j}$. The force $F_{i j, \omega}=-d W_{i j} / d \omega$ exerted in the direction of the displacement $\omega$ acts in such a way as to compress the outer island. However, this force is counteracted by $F_{j j, \omega}=-d W_{j j} / d \omega$, which acts to expand the outer island in both equilibrium branches. As a result, Fig. 18 shows that the symmetric equilibrium at $\omega=0^{\circ}$ is stable under a tilting displacement for the assumed initial conditions. A non-symmetric filament produced in the unstable high-energy branch proceeds to the stable position at $\omega=0^{\circ}$ (of course, the strongly compressed island may be subject to other MHD instabilities). The fold in the 


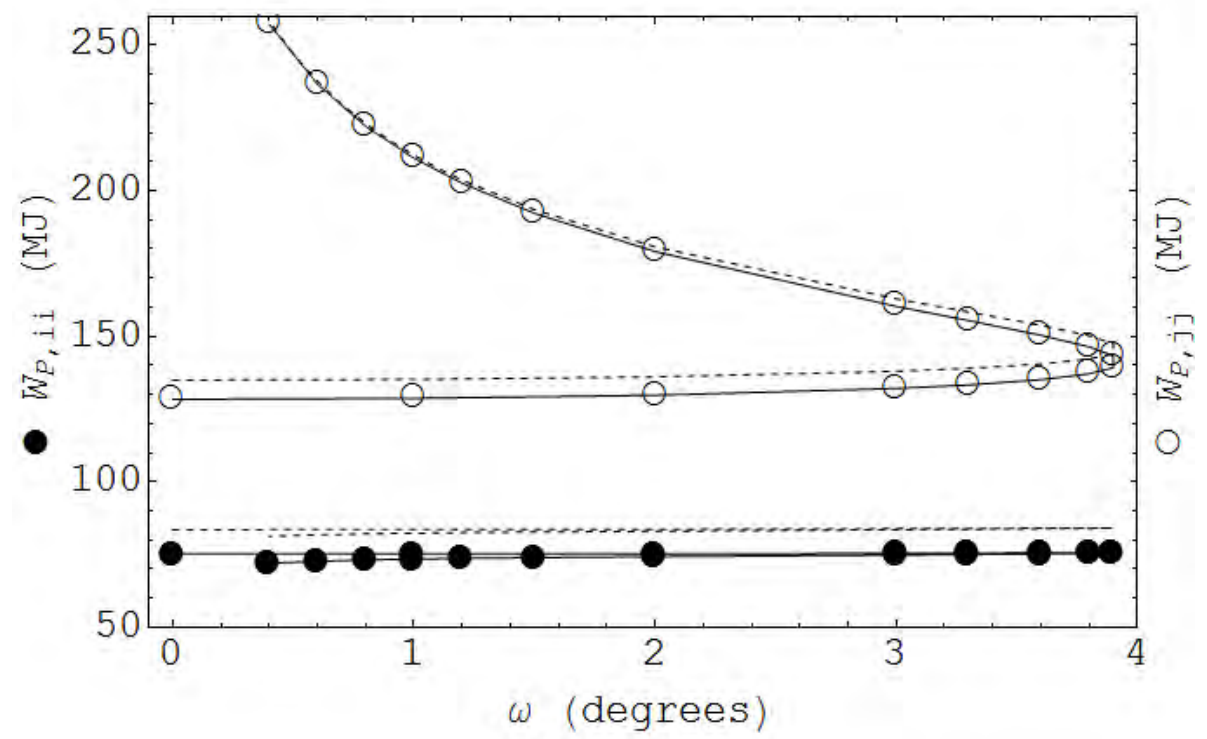

Figure 12: Poloidal self magnetic energies stored in the islands. The dashed lines correspond to the large-aspect-ratio approximation given in the text.

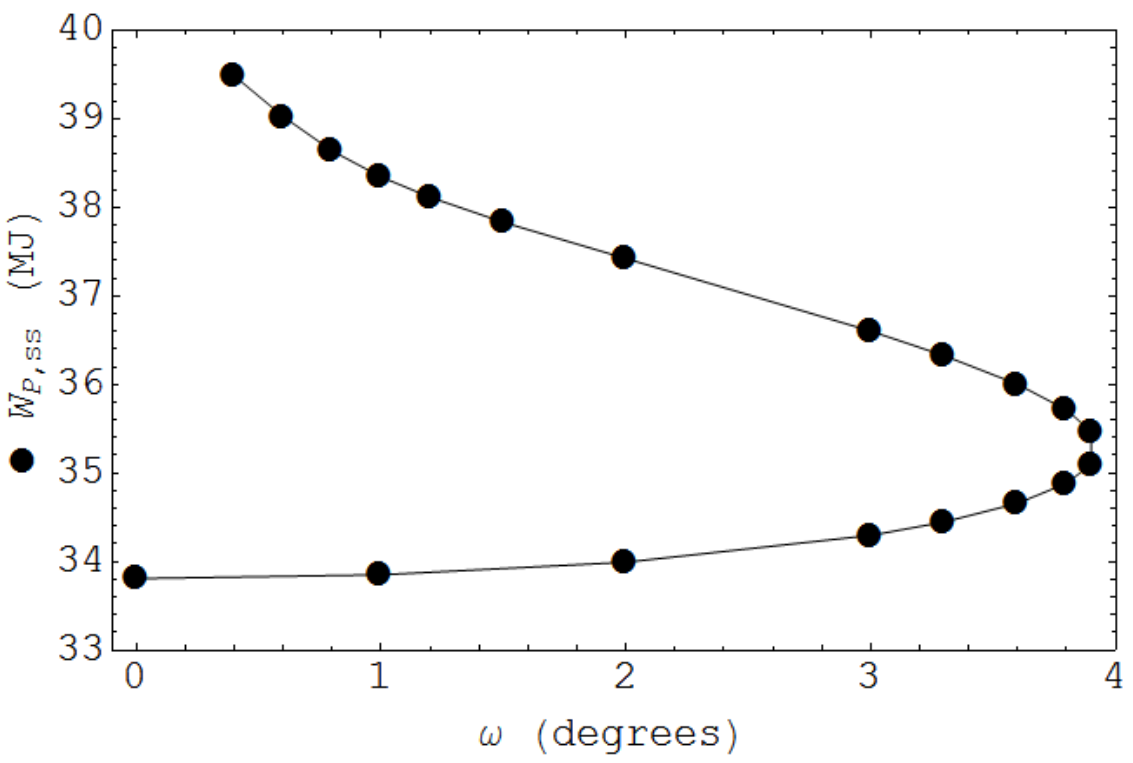

Figure 13: Poloidal self magnetic energy stored in the shell. 


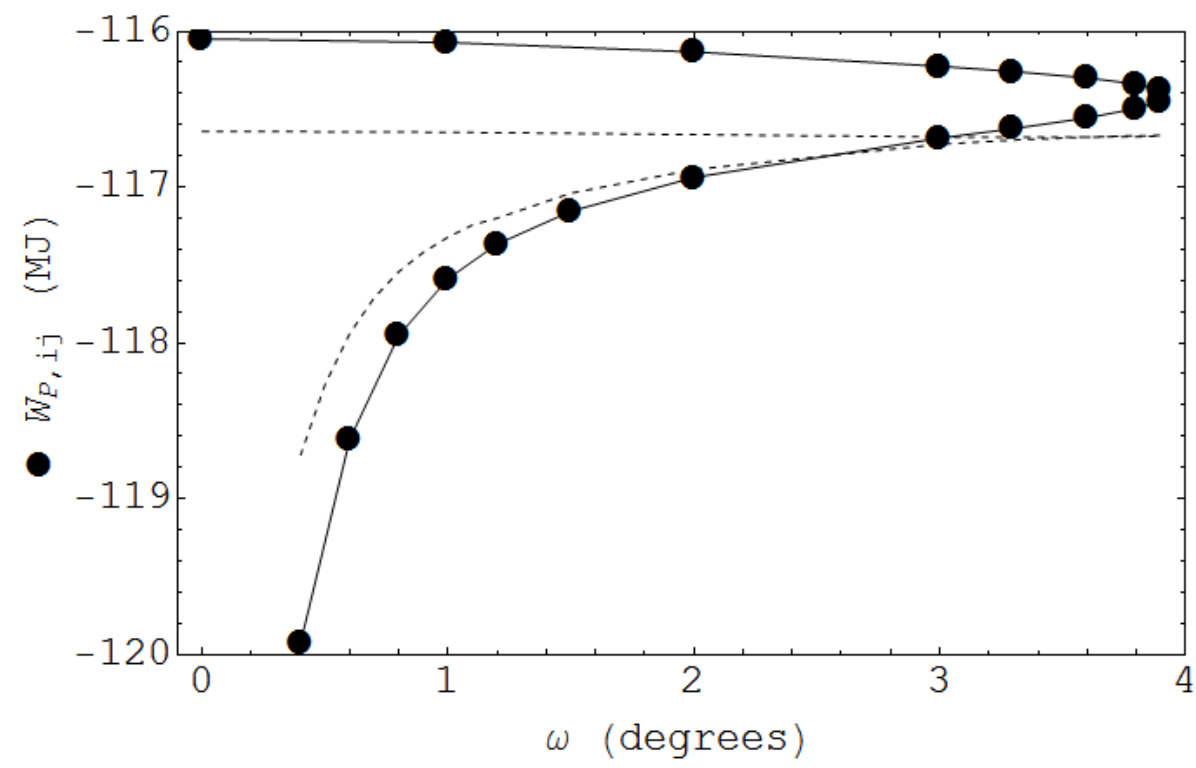

Figure 14: Poloidal magnetic field energy due to the mutual interaction between islands. The dashed line corresponds to the filaments approximation given in the text.



Figure 15: Poloidal magnetic field energies due to mutual interaction between the islands and the shell. 


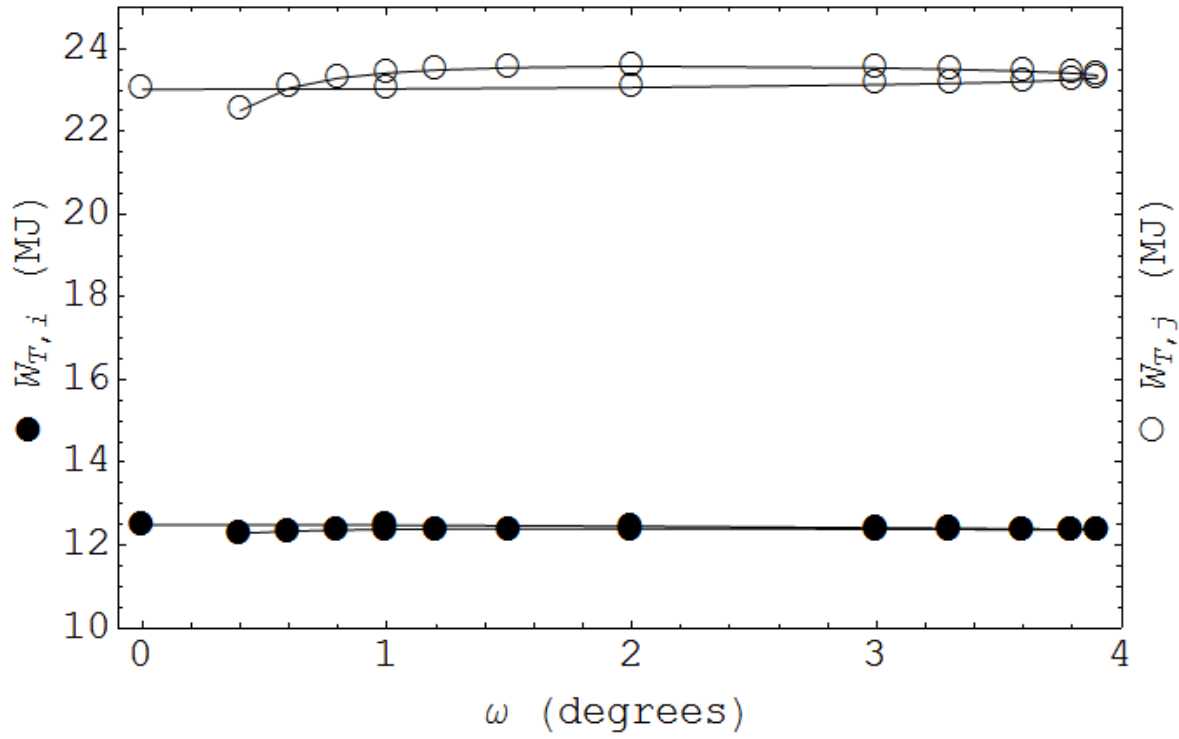

Figure 16: Toroidal magnetic field energies stored in the islands.

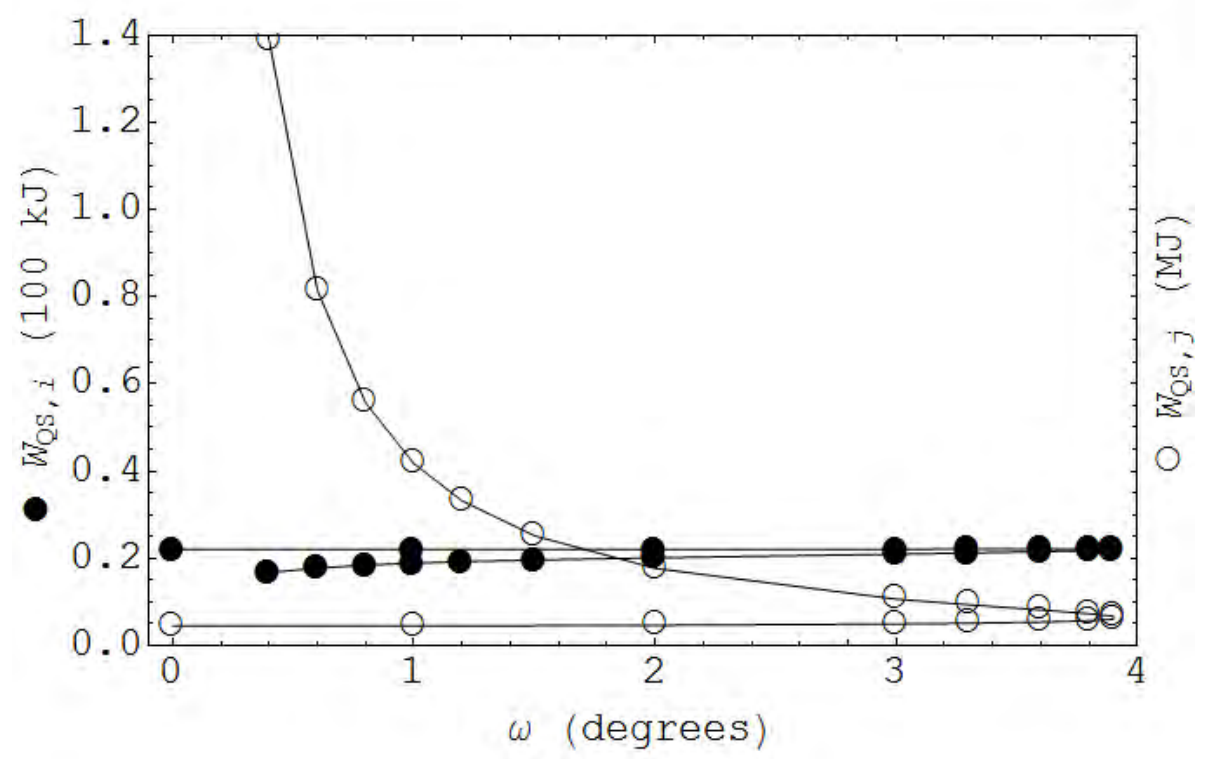

Figure 17: Quasistatic energies stored in the islands. Note the change of scale between the two islands. 


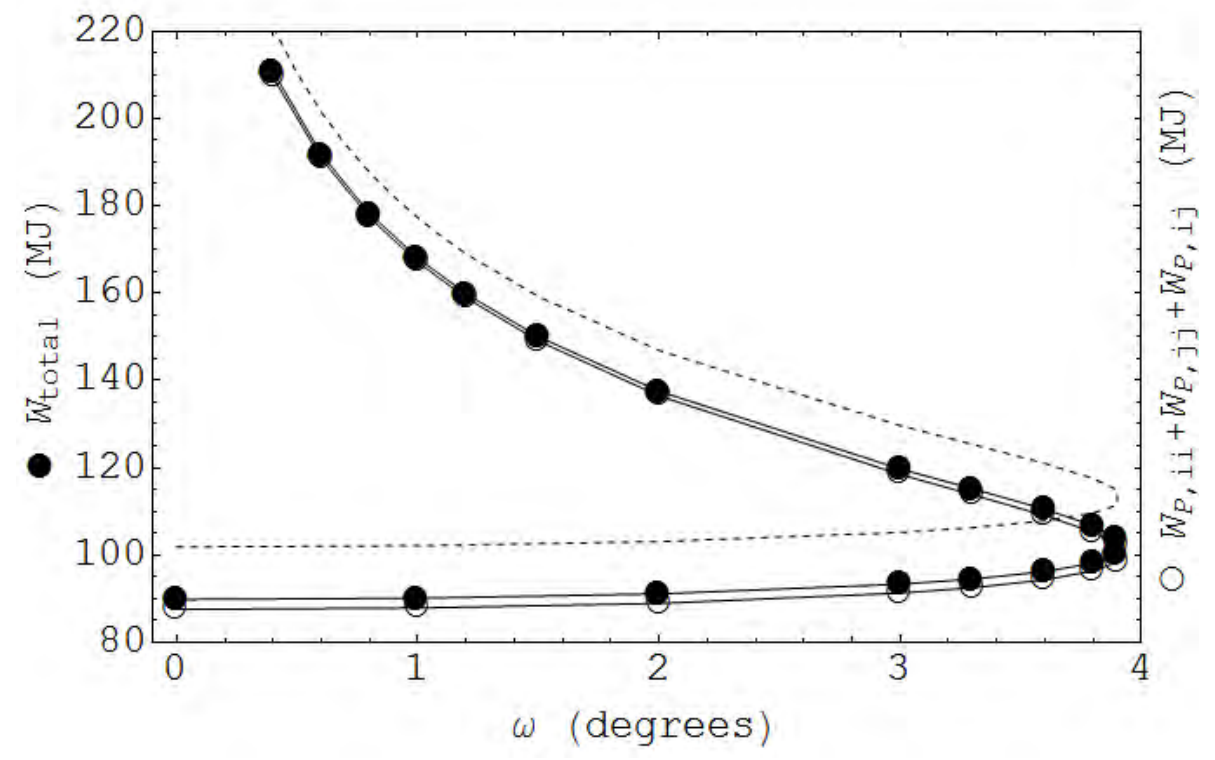

Figure 18: Total energy stored in the islands-shell system and dominant energy terms contributions. The dashed line corresponds to the combined large-aspect-ratio and filaments approximations given in the text.

equilibrium at the critical point $\omega \cong 3.9^{\circ}$ allows a continuous transition between the two equilibrium branches.

The energy terms associated with the external equilibrium are not included in the above analysis. In general, the energy balance of the entire system may be written in the form

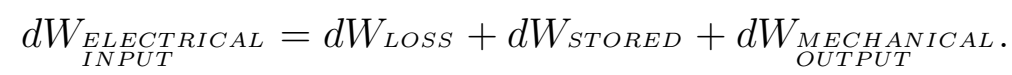

Since losses are neglected and there is no mechanical energy exchange with the exterior of the system, any change in the stored energy, which corresponds to the change in the total energy defined previously, is compensated by electrical energy feed by or into the external coils. In this paper it is assumed that the external coils system constitutes an infinite source or sink of electrical energy. The situation may differ in an externally controlled system as well as in the presence of heat losses (for example, in the outer tokamak plasma region/shell).

\section{Discussion}

In summary, the method presented makes it possible to investigate in a relatively simple way the equilibrium of complex configurations of non-nested flux surfaces. The method was applied to study the equilibrium of a pair of nonsymmetric islands contained by an ideal shell, which models the core of a tokamak with strong toroidal current reversal. Both the containing shell and the islands flux surfaces were described using spectral representations with distinct poloidal angles for either the shell or each simply connected region. With a reduced set of Fourier coefficients the internal equilibrium of each island can be solved effectively by the variational moments method. For simplicity, a Taylor series expansion of the variational moments solution to fourth-order in the radial coordinate has been used in this work. This series solution is adequate for the large-aspect-ratio 
configuration of the islands, although the spectral representations are not limited in the aspect ratio. Furthermore, the method uses the equivalent surface current density description in the internal (non-vacuum) flux-surfaces. This gives a straightforward formulation to the interaction between the different regions in terms of line integrals on the boundaries. Then, the equilibrium of the islands-shell system can be determined by matching moments of the Dirichlet boundary conditions. It is possible to satisfy as many moment equations as the number of unknown spectral coefficients on the islands boundaries. Also, the Dirichlet condition determines the surface current density distribution on the containing shell. At last, the currents in the external coils system were determined applying the Neumann boundary condition on the containing shell.

The proposed method, using spectral representations and equivalent surface current densities, leads naturally to a circuit-like approach to the equilibrium problem. The analytic expressions made available by the spectral representations simplify the computation of the stored energy terms, providing a tool for the analysis of the MHD stability by means of the energy principle. In this way it has been shown, for a class of tilting displacements, that the pair of symmetric islands in strong toroidal current reversal conditions in a tokamak core are stable. Moreover, the analysis has shown the occurrence, possibly for the first time, of a clear bifurcation in the MHD equilibrium. A very interesting result is the existence of two specular, nonsymmetric filamentary equilibrium solutions, separated by a branch cut, that may evolve into a large symmetric island through a tilting motion.

The present work concentrated on strong toroidal current reversal configurations, appropriate to $\mathrm{AC}$ tokamaks. In a future development both the dynamics of the $\mathrm{AC}$ regime and the small-islands weak-reversal configurations expected in the current-hole regime will be examined. However, firstly it may be necessary to obtain a spectral representation for flux surfaces in an annular region, improving the model of the outer plasma region.

Acknowledgment: This work was initiated during a visiting scientist contract of G.O.L. with the Centro de Fusão Nuclear, Associação Euratom-IST.

\section{References}

[1] Mitarai O., Wolfe S.W., Hirose A. and Skarsgard H.M. "Stable AC tokamak discharges in the STOR-1M device" Nucl. Fusion 27, 604-608, 1987

[2] Yang X., Jiang D., Li W., Han G., Wang L., Qi X., Feng C., Li Z. and Zheng S. "Alternating current operation with multicycles in the CT-6B tokamak" Nucl. Fusion 36, 1669-1678, 1996

[3] Cabral J.A.C., Fernandes H., Figueiredo H. and Varandas C.A.F. "Operation of the tokamak ISTTOK in a multicycle alternating flat-top plasma current regime" Nucl. Fusion 37, 1575-1581, 1997

[4] Li J., Luo J., Wang S., Fu P., Shen B., Liu F., Wan B., Shan J., Xu G., Huang J., Yu J., Hu J., Yuan Q., Hu Y. and HT-7 Team "Quasi-steady-state AC plasma current operation in HT-7 tokamak" Nucl. Fusion 47, 1071-1077, 2007

[5] Hawkes N.C., Stratton B.C., Tala T., Challis C.D., Conway G., DeAngelis R., Giroud C., Hobirk J., Joffrin E., Lomas P., Lotte P., Mailloux J., Mazon D., Rachlew E., Reyes-Cortes S., Solano E. and Zastrow K-D. "Observation of zero current density in 
the core of JET discharges with lower hybrid heating and current drive" Phys. Rev. Lett. 87, 115001-4, 2001

[6] Fujita T., Oikawa T., Suzuki T., Ide S., Sakamoto Y., Koide Y., Hatae T., Naito O., Isayama A., Hayashi N. and Shirai H. "Plasma equilibrium and confinement in a tokamak with nearly zero central current density in JT-60U" Phys. Rev. Lett. 87, 245001-4, 2001

[7] Huang J., Yang X., Zheng S., Feng C., Zhang H. and Wang L. "The plasma current profile during current reversal in AC operation of the CT-6B tokamak" Nucl. Fusion 40, 2023-2029, 2000

[8] Fujita T., Suzuki T., Oikawa T., Isayama A., Hatae T., Naito O., Sakamoto Y., Hayashi N., Hamamatsu K., Ide S. and Takenaga H. "Current clamp at zero level in JT-60U current hole plasmas" Phys. Rev. Lett. 95, 075001-4, 2005

[9] Rodrigues P. and Bizarro J.P.S. "Tokamak equilibria with toroidal-current reversal in the plasma core consistent with experimental data" Phys. Rev. Lett. 99, 125001-4, 2007

[10] Martynov A.A., Medvedev S.Yu. and Villard L. "Tokamak equilibria with reversed current density" Phys. Rev. Lett. 91, 085004-4, 2003

[11] Wang S. "Theory of tokamak equilibria with central current density reversal" Phys. Rev. Lett. 93, 155007-4, 2004

[12] Wang S. and Yu J. "An exact solution of the Grad-Shafranov-Helmholtz equation with central current density reversal" Phys. Plasmas 12, 062501-5, 2005

[13] Yu J., Wang S. and Li J. "Current reversal equilibrium configurations in the alternating-current operation of tokamaks" Phys. Plasmas 13, 054501-3, 2006

[14] $\mathrm{Hu}$ Y. "Tokamak plasma equilibria with a zero total toroidal current" Phys. Plasmas 15, 022505-10, 2008

[15] Rodrigues P. and Bizarro J.P.S. "Grad-Shafranov equilibria with negative core toroidal current in tokamak plasmas" Phys. Rev. Lett. 95, 015001-4, 2005

[16] Ludwig G.O. "Direct variational solutions of the tokamak equilibrium problem" Plasma Phys. Control. Fusion 39, 2021-2037, 1997

[17] Rodrigues P. Private communication, 2010

[18] Lao L. L., Hirshman S. P. and Wieland R. M. "Variational moment solutions to the Grad-Shafranov equation" Phys. Fluids 24, 1431-41, 1981

[19] Ludwig G.O., Del Bosco E., Ferreira J.G. and Berni L.A. "Simulation of eddy currents in spherical tokamaks" Nucl. Fusion 46, S629-S644, 2006

[20] Zhakarov L.E. "Numerical methods for solving some problems of the theory of plasma equilibrium in toroidal configurations" Nucl. Fusion 13, 595-602, 1973

[21] Breslau J.A., Jardin S.C. and Park W. "Simulation studies of the role of reconnection in the "current hole" experiments in the Joint European Torus" Phys. Plasmas 10, 1665-1669, 2003 